\title{
6. Anhang: Beschreibungen von Videos und Filmsequenzen
}

\section{1 netspanner (2011): Man films his own death in syria protest (Kapitel 1.1)}

Große graue Pixel in unterschiedlichen Farbabstufungen füllen den Rahmen. Der obere Rand ist von einer hellen, fast weißen Pixel-Linie durchschnitten. Darüber liegen graue Pixel in helleren Farbnuancen. Die Einstellung schwenkt schnell nach oben und die grauen Pixel werden als eine Betonbrüstung erkennbar, hinter der der obere Teil eines Fensters mit einem gelben, zugezogenen Rollo an einer grauen Gebäudewand sichtbar ist. Links oberhalb des Fensters ist undeutlich ein Kasten zu erkennen, der die Außeneinheit einer Klimaanlage sein könnte. Die Kamera schwenkt schnell nach links. Weitere Gebäudefragmente eines Wohnviertels kommen ins Bild: Balkone mit behangenen Wäscheleinen; Baumkronen, die zwischen den Häuserzeilen emporragen; Fenster, bei denen die braunen Rollos am helllichten Tag zugezogen sind. Ein dumpfer, hohler Sound ertönt: Es sind entfernte Schüsse, die im Echo der Gebäude widerhallen. Eine aufgeregte männliche Stimme spricht in schnellem Tempo auf Arabisch, während die Kamera unruhig weitere Gebäudefragmente einfängt und mal Dächer, mal Fassaden von Wohnkomplexen, mal Balkone zeigt. Der Mann hört auf zu sprechen. Er atmet schwer. Sein Atem oder der Wind verfängt sich im Mikrofon der Kamera. Ein entferntes Pfeifen und das hallende Rufen eines Mannes ertönen. Die Kameraführung ist unruhig und verwackelt. Die Betonbrüstung kommt schräg von unten wieder ins Bild. Nun ist deutlich ein Stimmengewirr einer Menschenmenge zu hören, aus der hin und wieder einzelne Stimmen und Schreie hervortreten. In direkter Nähe zur Kamera ist mindestens eine weitere Männerstimme zu hören. Der Filmende scheint auf die andere Seite des Balkons zu laufen, denn das Bild wird für einen Augenblick noch unruhiger. Die Kamera filmt für einen kurzen Moment nach unten in die Häusergasse hinein und schwenkt dann auf den Boden des Balkons, der von der Sonnenreflexion grelles Licht zurückwirft und die Aufnahme überlichtet. In der Überlichtung lassen sich die Umrisse von Mauersteinen ausmachen, während die Kamera unruhige und zu nahe Bilder des Balkons produziert. Dann verschwimmt das Bild zu grauweißen 
Schatten, während laute Schüsse im Hintergrund und das Knacken des Mikrofons $\mathrm{zu}$ hören sind. Eine undeutliche Bewegung findet im Bild statt, unterbrochen von einzelnen Bildern des strahlend blauen Himmels. Entfernte hallende Rufe sind zu hören und werden kurzzeitig von Bewegungsgeräuschen an der Kamera überlagert. Im nach links gekipptem Winkel ist der obere Teil eines hohen Wohnhauses vor blauem Himmel zu sehen. Mit einem weiteren Bewegungsgeräusch stellt sich das Bild wieder gerade und fährt nach links unten zu dem Fuß eines Wohngebäudes mit gelber Balkonbrüstung. Die entfernten Rufe werden von lauteren Schüssen überlagert. Die Kamera reagiert mit unruhigen Bildern des Hochhauses und fängt dann wieder den Fuß des Wohngebäudes ein. Hinter der Hausecke, dicht an der Gebäudemauer und im Schatten des Gebäudes, ist ein Paar Beine zu erkennen, das sich mit langsamen Schritten auf die Kamera zu bewegt. Im Hintergrund ist wieder das Stimmenrauschen der Menschenmenge mit vereinzelten lauten Rufen zu hören. Das Bild bleibt unruhig, reißt immer wieder ab und zeigt Fragmente des Himmels, des Gebäudes und der Straße mit parkenden Autos. Mit weiteren Bewegungsgeräuschen kommt wieder der Fuß des Wohngebäudes ins Bild. Aus dem Schatten heraus hat sich eine schemenhafte Gestalt in einem khakifarbenen Overall bis zur Hausecke vorgearbeitet. Sie hält ein Gewehr in Bereitschaftsposition. Sie bringt das Gewehr in Anschlag. Das Bild bleibt verwackelt, aber auf die Gestalt fixiert. Dann verliert es kurz die Gestalt mit Gewehr und zeigt ein weiteres Fragment des Wohngebäudes, während im Hintergrund aufgeregte Rufe und Bewegungsgeräusche an der Kamera zu hören sind. Das Bild findet die Gestalt in schussbereiter Position wieder. Ein alles übertönender Schuss erschallt und die Aufnahme fällt völlig aus dem Rahmen: ein helles, fast grelles Bild gefolgt von einem rotkarierten Bild, wird begleitet von knackenden, dumpfen Tönen als würde etwas auf den Boden fallen. Dann folgen wieder helle, zunächst weiße, danach gelbliche Bilder und schließlich weißgrauer Stillstand. Auch auf der Tonebene herrscht einige Sekunden lang Stille bis eine wimmernde Männerstimme und eine weitere, aufgeregt auf Arabisch sprechende Männerstimme lauterwerdend ertönen. Nach wenigen Sekunden folgen schreiende Ausrufe einer weiteren Männerstimme. Erneut hallen Schüsse im Hintergrund. Der Ton reißt noch einmal ab. Dann sind für knapp 20 Sekunden immer wieder zwei Männerstimmen zu hören, die auf Arabisch miteinander reden, während weiterhin Schüsse im Hintergrund ertönen. Das Video endet unvermittelt.

\subsection{MikeTysonFan1011 (2010): Photographer Leonardo Henrichsen filming his death (Kapitel 3.1.1)}

Die schwarzweiße Aufnahme setzt mit einer davonstürzenden Menschenmenge in einer halbtotalen Einstellung ein, die eine große Straße bei Tageslicht entlangläuft. 
Die Straße ist am linken Bildrand von hohen Gebäuden und Straßenlaternen gesäumt. Die Bildqualität ist körnig verschwommen, so dass die Gesichter der Menschen nicht klar zu erkennen sind. Die Kamera befindet sich auf gleicher Höhe mit den Menschen auf der Straße. Die Menschen laufen auf die Kamera zu und an ihr vorbei. Die Kameraführung ist unruhig. Auf der Tonebene ist ein stetes Gewehrfeuer nicht-sichtbarer Waffen zu hören. Ansonsten gibt es keine Geräusche: weder die rennenden Schritte der Menschen noch die Schreie oder Ausrufe sind hörbar. Ein Militärjeep taucht im Hintergrund auf einer entfernten Straßenkreuzung auf und fährt quer zur Blickrichtung der Kamera auf die gegenüberliegende Straßenseite. Die Kamera schwenkt mit der Fahrtrichtung des Jeeps und zoomt heran. Das Bild wird unruhiger und verliert kurzzeitig den Fokus. Auch die Fahrgeräusche des Jeeps sind nicht zu hören. Der Jeep parkt am rechten Straßenrand. Die Ladefläche ist mit mehreren bewaffneten Soldaten besetzt. Der Fahrer und einige der Soldaten verlassen den Jeep. Die Kamera zoomt wieder heraus, um den Straßenzug mit den davonlaufenden Menschen einzufangen und nur wenige Sekunden später wieder auf den Jeep zu zoomen. Das Gewehrfeuer wird weniger. In kurzen Feuerpausen sind im Hintergrund undeutliche Stimmen und auch eine deutlichere Männerstimme zu vernehmen, die auf Spanisch etwas sagt. Zwei der aus dem Jeep ausgestiegenen Soldaten entfernen sich mit zügigen Schritten und Gewehren in Anschlagsposition vom Jeep. Zwei weitere bleiben mit ihren Gewehren in unmittelbarer Nähe des Jeeps stehen. Ein Off-Kommentar kommentiert auf Spanisch die Situation, während im Hintergrund weiterhin vereinzelte Schüsse zu hören sind. Auf der Ladefläche verharren weitere fünf Soldaten, teilweise mit Gewehren in Anschlag. Ein Passant eilt zwischen Kamera und Jeep vorbei. Am rechten Bildrand ist der hintere Teil eines PKWs zu sehen, der am Straßenrand hinter dem Militärjeep abgestellt ist. Auf einmal erscheint ein halbrunder Riss in schwarzen, sich verzweigenden Linien am linken Bildrand, der auf dem Bild liegt. Die Kamera zoomt erneut heraus. Der Riss verweilt unbeweglich auf dem Bild. Auf der Straße befinden sich nur noch wenige Menschen, die sich die Straße hinunter - allerdings ruhigeren Schrittes - bewegen. Links kommt eine Eingangstreppe ins Bild, über die einige Menschen ein Gebäude betreten. Andere wenden sich um, während sie die Straße hinunter an der Kamera vorbeilaufen oder die Straße überqueren. Der Ton geht zu einem leisen, unbeständigen Pfeifen und noch leiseren Stimmgeräuschen über. Nur noch einzelne Schüsse sind zu hören. Das Gewehrfeuer stoppt für mehrere Sekunden. Ein Schnitt. Der Riss auf dem Bild ist verschwunden. Es befinden sich keine Passanten mehr auf der Straße. In einer Totalen sind der am Straßenrand geparkte Militärjeep und ein weiteres geparktes Auto am rechten Bildrand zu sehen. Zwischen den Fahrzeugen stehen verstreut Soldaten. Auf dem Boden vor ihnen liegt ein dunkler, undeutlicher Haufen. Die Kamera zoomt schnell bis zur halbnahen Einstellung heran. Das Bild ist verwackelt und zuerst unscharf. Dann stellt sich das Bild scharf und eine auf dem Boden liegende, sich wiederaufrich- 
tende Person wird erkennbar, neben der ein Militär mit gezogener Handfeuerwaffe steht. Er ruft ihr etwas zu und macht eine scheuchende Handbewegung. Ein weiterer Militär mit schussbereitem Gewehr kommt ins Bild. Im Hintergrund sind noch vier weitere Soldaten zu sehen. Erneut ertönt stetiges Gewehrfeuer nicht-sichtbarer Waffen, ohne das weitere Umgebungsgeräusche zu hören sind. Der Soldat mit Handfeuerwaffe dreht sich zu dem hinter ihm stehenden Soldaten um, gestikuliert mit der freien Hand und geht mit zur Kamera gerichtetem Rücken ein kurzes Stück auf die Soldaten zu. Das Kamerabild wackelt kurzzeitig. Dann wendet sich der Soldat zur Kamera um und richtet seine Waffe auf sie. Ein einzelner Schuss sticht aus dem Gewehrfeuer heraus. Das Bild verwackelt erneut, wird unschärfer und verliert einen Moment den Fokus, bis es den Soldaten in einer halbtotalen Einstellung wieder einfängt, wie er sich von der Kamera entfernt und am rechten Bildrand aus dem Bild verschwindet. Die Kamera fokussiert nun einen anderen Soldaten, der in direkter Nähe zum Militärjeep sein Gewehr auf die Kamera richtet. Auf der Ladefläche des Jeeps stehen drei weitere Soldaten. Aus dem intensiver werdenden Gewehrfeuer stechen erneut zwei Schüsse heraus. Der letzte ertönt als der Soldat sein Gewehr bereits wieder senkt und sich von der Kamera abwendet. In der linken oberen Ecke legen sich wieder schwarze Risse über das Bild. Der Soldat bewegt sich langsam in Richtung Militärjeep. Die Kamera zoomt etwas weiter nach links auf die Ladefläche des Jeeps. Ein Soldat, der auf der Ladefläche steht, hat sein Gewehr auf die Kamera gerichtet und feuert mit hörbarem Schuss. Er lädt das Gewehr nach. Vereinzelt legen sich kleine schwarze Flecken über das Bild, um im nächsten Augenblick wieder zu schwinden. Das Bild beginnt heraus zu zoomen, schwankt und fällt plötzlich aus dem Rahmen: Eine blanke, graue Oberfläche füllt das Bild mit dunkler- und wieder hellerwerdenden Schatten und Formen aus. Im Hintergrund ist weiterhin das stete Gewehrfeuer mit leisen Stimmgeräuschen zuhören. Der Filmausschnitt endet.

\section{3 hoyartil (2011): Homs- Basel Sayyed's last camera footage before his death by Assad's thugs. 22-12-2011 (Kapitel 3.2)}

Lautes Maschinengewehrfeuer vor einem unscharfen, überbelichteten und leicht nach links gekipptem Bild. Der linke Bildrand ist durch ein gestricktes Kleidungsstück, vermutlich der Pullover oder die Jacke des Filmenden, abgeschnitten. Im freien Bildfeld sind zwei schattenhafte Gestalten zu sehen. Ein Insert erscheint im Bild: »Homs The 24 years old martyr Basel Alsayyed, got targeted and shot in the head by Assad's thugs on 22-12-2011«. Lautes, dröhnendes Maschinengewehrfeuer ist $\mathrm{zu}$ hören. Im freien rechten Bildbereich stürzen abgeschnittene Gestalten davon und verschwinden links aus dem Bild. Die Lichtverhältnisse werden ausgeglichener. Die Aufnahme ist in Bewegung. Nur der durch die Kleidung des Filmenden 
abgeschirmte Bereich auf der linken Seite bleibt statisch. Ein Paar Beine eilt links aus dem Bild bevor rötliche Asphaltplatten einer Straße den rechten Bildbereich ausfüllen. Das Bild bleibt für mehrere Sekunden statisch auf dem Asphalt fixiert. Kaum merkbare Bewegungen des Bildes suggerieren das Filmen mit einer Handkamera. Die Kamera scheint eng am Oberkörper des Filmenden zu liegen, halb abgeschirmt von der Umgebung. Das Maschinengewehrfeuer hält an. Undeutliche Ausrufe und Schreie von Menschen treten zwischendurch daraus hervor. Die Beschriftung des Inserts wechselt: »because he insisted to document Assad's crimes against Baba Amr, Homs by his camera.« Das Bild gerät erst leicht, dann stärker in Bewegung ohne das Motiv zu wechseln. Eine aufgeregte Männerstimme in unmittelbarer Nähe zur Kamera ruft dreimal: »Allah Akbar!« Das Bild wird unruhig, beginnt zu schwanken und kippt nach links. Der Filmende scheint mit der Kamera zu Boden zu fallen. Seine Kleidung füllt das gesamte Bild aus, das sich in einer Linksrotation dreht. Das Insert wechselt erneut: »He was shot when trying to document the shooting of thugs on innocent people from a checkpoint. « Das Bild überlichtet kurz. Ein Knistern ertönt während die >Allah-Akbar<-Ausrufe und das Maschinengewehrfeuer anhalten. Das Bild wird schwarz. Die >Allah-Akbar-Ausrufe verstummen und eine weitere, undeutliche Männerstimme ertönt. Im Insert liest sich: »The martyr died later of his wounds on 29-12-2011. "Aufgeregte Schreie und einzelne Ausrufe, dann ein anhaltendes Stimmengewirr und erneutes Maschinengewehrfeuer sind zu hören. Das Insert wechselt ein letztes Mal: »Glorified your will, your bravery, your devotion, brother.« Das Bild bleibt schwarz, während die Tonspur weiterläuft und sich das Geschehen dramatisch zu zuspitzen scheint: ein stetes >Allah-Akbar-Rufen, Pfiffe, Schreie, eine dumpfes Einschlag- oder Explosionsgeräusch, ein Klirren und Schüsse ertönen. Die Tonspur bricht unvermittelt ab und das Video endet.

\section{4 al-Jazeera English (2011): Syrian protesters capture own death on camera (Kapitel 3.3.1)}

Das Video setzt mit den verwackelten und verpixelten Bildern von Wohngebäudefragmenten ein, die den Beginn des netspanner-Videos kennzeichnen (vgl. 6.1). Nur wenige Bilder der verpixelten Betonbrüstung ganz zu Anfang des Videos sind abgeschnitten. Ein Voiceover einer weiblichen Stimme leitet im journalistischen Stil durch die Bilder:

A man stands filming from a balcony in the Syrian town of Homs. The voice says, it's July first and that someone is shooting on people for no reason. This is last Friday when Syria saw its biggest nationwide protest yet. Then the camera catches a man on the balcony at the building nearby. He moves forward and takes aim. 
We hear a voice saying: >The man has been hit by a bullet to the head. $<$ And then what could be the voice of the camerman saying: >Bullets. Bullets. Bullets.< (ebd.)

Aus der Mitte des netspanner-Videos wurden mehrere Sekunden Material herausgeschnitten, die die Bewegung des Filmenden auf dem Balkon wiedergeben. Ebenso wurde die Schusssequenz um Bilder gekürzt, in denen verwackelte Gebäudefragmente sichtbar sind und das Hantieren mit der Kamera hörbar ist. Die Gestalt mit dem Gewehr erscheint größer im Bildausschnitt als im netspanner-Video. Das Bild scheint nachträglich herangezoomt worden zu sein. Auch nach dem Schuss sind Schnitte zu erkennen: nach den Geräuschen der vermeintlich auf den Boden fallenden Kamera, folgen nur wenige Sekunden lang Bilder des weißgrauen Stillstands mit dem Wimmern und der aufgeregten Männerstimme im Hintergrund, bevor das Video mit einer Überblende zurück zur Schusssequenz wechselt, um den Todesschützen mit erhobenem Gewehr noch einmal in einer herangezoomten Totalen in einem Standbild zu zeigen (vgl. 6.1). Mit einer weiteren Überblende und den Worten des weiblichen Voiceovers »We cannot independently verify the authenticity of this video nor can we identify who is filming", geht das Video zu einem anderen Internetvideo über. Am hellichten Tag ist in einer Totalen aus der Vogelperspektive eine größere, verstreut umherlaufende Ansammlung von Menschen auf einer Straße in einem Wohnviertel, die von hohen Gebäuden und vereinzelt parkenden Autos gesäumt ist, zu sehen. Der rechte Bildrand ist durch einen Teil einer Gebäudefassade abgeschnitten. Das Bild schwankt leicht und ist unruhig. Das Voiceover fährt fort: »But another video, which was posted online from the same day and location in Homs, seems to tell the same story."Stimmengewirr und Schüsse gefolgt von entfernten Schreien sind zu hören. Die Menschen beginnen vom oberen Bildrand die Straße hinunterzurennen. Immer mehr Menschen strömen die Straße hinab. »The Syrian government makes it very difficult for journalists to approve from inside the country. « Die Einstellung beginnt stärker zu schwanken. Ein Geländer, vermutlich eines Balkons, ist kurz am unteren Bildrand dicht an der Kamera zu sehen. Der Filmende scheint sich zu bewegen. »So we rely on videos uploaded to the Internet...« Die Aufnahme wird wieder ruhiger als der Strom der Menschen vom oberen Bildrand nachlässt. "...or sent to us by citizens to get an idea of what is happening inside Syria." Auf der Straße befinden sich nur noch vereinzelt davonlaufende Menschen. "At times it's difficult to establish precise facts. «Etwa in der Bildmitte bildet sich eine kleine Menschentraube. »But videos like these appear to show the systematic...«, drei Männer bleiben zurück und tragen eine anscheinend angeschossene, am Boden liegende Person zwischen zwei am Straßenrand parkende Autos, die sich am linken unteren Bildrand befinden. Plötzlich läuft eine einzelne Person von den parkenden Autos aus in die entgegensetzte Richtung diagonal zur anderen Straßenseite. Sie befindet sich alleine auf der Straße und macht mit dem rechten Arm eine Wurfbewegung in Richtung eines 
unsichtbaren Ziels im rechten Off. »...brutal crackdown on anti-government protesters. « Erneut fallen Schüsse. Die Person beginnt zu taumeln und fällt regungslos zu Boden. Ein nahes »Ohhh...« einer Männerstimme ist zu hören. Im Hintergrund ertönen aufgeregte Frauenrufe. Das Voiceover endet mit: »Stephanie Dekker. alJazeera. "Das Geländer taucht wieder vom unteren Bildrand auf und lässt das Bild fast komplett dunkel werden, bevor die Videoreportage in einer Überbelichtung endet.

\subsection{EAU ARGENTÉE (2013) (F, R: Mohammed/Bedirxan): Dimanche \& Lundi (Kapitel 3.4.1)}

Nach der Titeleinstellung Dimanche (dtsch.: Sonntag) in weißer arabischer und lateinischer Schrift auf schwarzem Hintergrund folgt eine statische Aufnahme eines Fotos eines Jungen und eines Mädchens in einer halbtotalen Einstellung. Der Junge ist ungefähr sieben oder acht Jahre, das Mädchen ungefähr fünf oder sechs Jahre alt. Der Junge steht mit seinem roten Fahrrad in seitlicher Position zur Kamera und lächelt freundlich in die Kamera. Er trägt eine Jeanshose, eine Jeansjacke und dunkle Schuhe. Seine dunklen Haare sind kurz geschnitten. Mit der einen Hand umfasst er den Griff des Lenkers, mit der anderen die Mitte des Lenkers. Mit einem Bein steht er fest auf dem Boden, mit dem anderen mit hoch angewinkeltem Knie auf einem Pedal. Links neben ihm steht das Mädchen, ebenfalls freundlich in die Kamera lächelnd. Auf dem Arm hält sie, fest an sich gedrückt, ein Kätzchen mit weißem Fell und schwarz gezeichneten Ohren. Ihre dunklen, langen Haare sind zu einem seitlichen Zopf geflochten. Sie trägt eine rosafarbene Jacke mit weißen Bündchen, eine dunkelblaue Hose mit abgesetzten Taschen und dunkle Schuhe. Die beiden Kinder stehen auf einer Straße zwischen hohen, betonfarbenen Häuserzeilen. Teilweise sind die Wände mit Graffiti bemalt. Zwischen den Häuserzeilen dringt die Sonne hindurch und malt einen Lichtschatten durch die Speichen des Fahrrads auf den Asphalt. "Yassin und Mariam«, sagt Bedirxans Stimme auf Kurdisch im hinzugefügten Audio (mit deutschem Untertitel), das von einem deutschen Untertitel begleitet wird. Gleichzeitig ist ein leises Stimmengewirr zu hören. Schnitt: Titeleinstellung Lundi (dtsch.: Montag) in weißer arabischer und lateinischer Schrift auf schwarzem Hintergrund. Ein weiteres Foto der beiden Kinder in einer Nahaufnahme in statischer, stummer Einstellung folgt. Ihre Körper sind völlig in glänzenden, grünen Stoff gehüllt, der nur ihre Gesichter frei lässt. Oberhalb ihrer Köpfe ist der Stoff jeweils mit einem weißen Band zusammengebunden. Sie liegen mit ihren Rücken regungslos auf einem dunklen, metallenen Untergrund, der an die Ladefläche eines Fahrzeuges erinnert. Die Lichtverhältnisse sind dunkel und erwecken den Eindruck, dass das Foto nicht unter freiem Himmel aufgenommen wurde. Links blickt das Mädchen mit leeren Augen nach oben aus 
dem Bild heraus. Ihr Mund ist leicht geöffnet. Ihr Kopf ist leicht nach links geneigt. Rechts liegt der Junge. Sein Gesicht ist gerade zur Kamera gerichtet. Seine Augen sind geschlossen. Sein Mund ist ebenfalls leicht geöffnet. Bedirxans Stimme sagt aus dem Off (mit deutschem Untertitel): »Mariam und Yassin«. Schnitt: Szenenwechsel. Eine Großaufnahme mit natürlichen Lichtverhältnissen von zwei mit arabischen Schriftzeichen bemalten Betonsteinen mit glatter Oberfläche. Eine dunkle Fuge fügt die Steine zusammen. Die Kamera schwenkt leicht nach unten. Der Rücken einer Hand kommt von unten ins Bild. Flach ausgetreckt streifen die Fingerspitzen über die Oberfläche des Steines. Im Synchronton sind ein schweres Atmen und leise Umgebungsgeräusche zu hören. Die Finger klopfen sanft, aber vehement auf die Oberfläche. Bedirxans, von Trauer gezeichnete Stimme ruft (mit deutschem Untertitel): »Yassin! Mariam!« Die Kamera schwenkt etwas weiter nach unten. Am unteren rechten Rahmen kommt ein schräg durch die Einstellung laufender bräunlicher Randstein ins Bild. Die Finger klopfen fester auf die Oberfläche. »Wacht auf!«, ruft Berdirxans Stimme (mit deutschem Untertitel). Die Hand stoppt das Klopfen und bewegt sich nach oben aus dem Bild, während die Kamera noch einen Augenblick statisch auf den Steinen verweilt.

\subsection{EAU ARGENTÉE (2013) (F, R: Mohammed/Bedirxan): Havalo, ich bin verwundet (Kapitel 3.4.1)}

Die Innenfläche einer Hand in extremer Detailaufnahme füllt den Bildrahmen völlig aus. Die Handlinien sind deutlich zu erkennen. Das Bild ist hell ausgeleuchtet. Im Synchronton sind hohle Fahrgeräusche wie im Innern eines Autos zu hören sowie dumpfe Stimmen. Das Bild wackelt leicht. Bedirxans, später zur Aufnahme hinzugefügte Stimme ruft (mit deutschem Untertitel): »Havalo [Kurdisch für >mein Freund , gemeint ist Mohammed; MM], ich bin verwundet.« Im Synchronton sagt Bedirxan mit heller, tragisch lachender Stimme (mit deutschem Untertitel): »Ich filme mich. Ich filme meine Verletzung.« Ein Mann spricht mit ihr (seine Worte werden nicht übersetzt). Weitere Stimmen sind nun deutlicher zu hören. Das Bild überbelichtet kurz, ohne dass sich die Einstellung der Handinnenfläche ändert. »Ich bin verwundet «, ruft Bedirxan (mit deutschem Untertitel) und kichert im Synchronton. Schnitt. Nahaufnahme einer länglichen Wunde am Oberschenkel. Die gedämpften und ausgegelichenen Lichtverhältnisse deuten daraufhin, dass die Aufnahme im Innern eines Gebäudes mit Deckenbeleuchtung erfolgt. Mit einer chirurgischen Zange und Nadel und Faden wird die Wunde von zwei Händen in weißen Latexhandschuhen genäht. Über der Wunde ist die blaue Hose aufgerissen. Das Bein liegt auf einer Bahre von unten nach oben senkrecht im Bildrahmen. Im Hintergrund ist das synchrone Stöhnen von Bedirxan zu hören. Schnitt. Die Kamera schwenkt unruhig über die linke Seite von Bedirxans Oberkörper hin- 
über zu ihrem Gesicht. Sie trägt eine rote Bluse, deren Ärmel bis zum Ellenbogen hochgekrempelt sind. In einer Großaufnahme liegt sie mit dem Rücken flach auf der Bahre. Ihre langen dunklen Haare sind hinter dem Kopf zusammengebunden. Sie singt mit sanfter Stimme. Schnitt. In einer Nahaufnahme wird das Nähen der Wunde an Bedirxans Oberschenkel gerade abgeschlossen. Ein lautes, hallendes Hupen, das von Außerhalb des Raumes zu kommen scheint, übertönt kurz Bedirxans Gesang. In der linken Bildecke kommt eine metallene, längliche Dose ins Bild, die mit geöffnetem Deckel auf der Bahre neben dem verwundeten Bein liegt und weitere chirurgische Instrumente enthält. Die mit weißen Latexhandschuhen bekleideten Hände verknoten die Fäden als eine männliche Stimme aus dem Off fragt (mit deutschem Untertitel): »Sag, wovon handelt dein kurdisches Lied?« Bedirxan verstummt. Die Kamera schwenkt wieder zurück zu ihrem Gesicht. In einer verwackelten Großaufnahme sagt sie (mit deutschem Untertitel): »Ich schwitze.« Sie streift sich mit der freien, linken Hand seitlich über die Wangen und atmet schwer aus. Die Augen hat sie dabei geschlossen. Im Hintergrund sind leise Stimmen zu hören. Dann hält sie die Kamera am ausgestreckten Arm über sich. Die Augen halb geöffnet, blickt sie rechts an der Kamera vorbei. In dieser Großaufnahme von ihrem Gesicht verweilt die Aufnahme wenige Sekunden, während die Bilder stumm in Zeitlupe wiedergegeben werden.

\subsection{EAU ARGENTÉE (2013) (F, R : Mohammed/Bedirxan) : Et le cinéma fut (Kapitel 3.4.2)}

Die Titeleinstellung mit weißer arabischer und lateinischer Schrift auf schwarzem Hintergrund zeigt: Et le cinéma fut. In der deutschen Übersetzung in gelber Schrift liest sich darunter: Und es ward Kino. Ein schwarzes Bild folgt für wenige Sekunden. Im Audio sind zuerst das Schreien eines Babys, dann ein heller Pfeifton und ein leises Wasserplätschern zu hören. Plötzlich wechselt die Aufnahme mit einem tiefen Trommelschlag zu Bildern von $\mathrm{zu}$ nahen und schnell wechselnden Einstellungen von Teilen von bekleideten Körpern, Schatten und Schemen. Die Bilder sind verwackelt und verpixelt, hell und zeitweise überbelichtet. Die Kamera scheint sich Draußen bei Sonnenschein zu befinden. Im Hintergrund ruft ein rhythmischer Chor mit den arabischen Worten (mit deutschem Untertitel): »Freiheit! Freiheit!« Schnitt. Wieder ein schwarzer Bildschirm mit weißer arabischer und gelber lateinischer Schrift: "Ich kann nicht filmen. Eine männliche Stimme spricht im verzerrten Ton einer Mikrofonaufnahme die vermutlich gleichen Worte auf Arabisch. Die Aufnahme wechselt mit einem Schnitt zu einer totalen Einstellung mit nach links gekipptem Stadtpanorama. Der untere Teil des Bildes ist mit abgeschnittenen Haarschöpfen gefüllt, die mit der horizontalen Ausrichtung des Bildes zu einer Protestmasse - vermutlich aufgenommen mit nach oben ausge- 
strecktem Arm - wird. Über der Protestmasse ist der blaue Himmel mit leichten weißen Wolken zu sehen. Die Bilder sind mal zu dunkel, mal zu hell. Die Autobelichtung der Kamera scheint sich während der Aufnahme den Lichtverhältnissen anzupassen. »Lasst unsere Kinder frei!« werden die Rufe der Protestmasse mit einer Einblendung in gelber Schrift am unteren Bildrand übersetzt. Schnitt. Vor schwarzem Hintergrund mit weißer arabischer Schrift liest sich: „Das ist mein erstes Mal«, während die männliche, verzerrte Stimme die vermutlich gleichen Worte auf Arabisch spricht. Der Ton der Protestmasse läuft leiser weiter. Ein Pfeifen ist zu hören. Schnitt. Wechselnde Aufnahmen von Protestchören in halbtotaler Einstellung in einer urbanen Umgebung in schlechter Videoqualität folgen. Die Menschen sind unterhalb des Oberkörpers vom unteren Bildrand abgeschnitten. Ein Mann im Vordergrund hält mit der rechten Hand ein weißes Blattpapier mit großen arabischen Worten in die Höhe. Dann fasst er es auch mit der linken Hand und hält es direkt in die Kamera, so dass das Blatt gut sichtbar im Bildrahmen erscheint. Kurzzeitig kommt dabei auch sein Gesicht frontal ins Bild. Mit gelber Schrift werden die Worte darunter übersetzt: NEIN ZUR VERHAFTUNG VON KINDERN. Im Hintergrund wird ein weiteres beschriftetes Blattpapier hochgehalten. Mit einer überbelichteten Einstellung wechselt das Bild zu Aufnahmen einer weiteren Protestmasse. Die Menschen füllen eine Straße mit hohen Wohngebäuden aus. Die Kamera filmt oberhalb der Köpfe die Straße hinunter. Die Bilder sind stark verwackelt und leicht verpixelt. Viele Menschen heben eine Faust in die Luft, während im Sprechchor gerufen wird. Am unteren Bildrand in gelber Schrift lautet die Übersetzung hierzu: »Freiheit, Freiheit!« Die Sprechchöre wechseln. Die Übersetzung lautet: »Das Volk will den Sturz des Regimes!« Schnitt. Eine weitere, verwackelte Aufnahme einer Protestmasse in städtischer Umgebung vor blauem Himmel mit leichten weißen Wolken. Im Vordergrund ragt eine ausgestreckte Hand in Richtung Himmel und bewegt sich rhythmisch zum Sprechgesang, der mit »Beendet den Ausnahmezustand! Freiheit, Freiheit!« übersetzt wird. Nach drei Sekunden folgt ein Schnitt und ein Bild der ausgestreckten Hand wiederholt sich mehrere Male. Schnitt. Mit dem Geräusch eines Luftausstoßes oder Ausatmens erscheint die stumme Aufnahme einer verstreuten Menge von Menschen in einer halbtotalen Einstellung auf einem urbanen Platz am helllichten Tag. Die Menschen werfen lange Schatten von einer abendlichen Sonne auf den Asphalt. In der subjektiven Kamera zeigt eine Hand mit ausgestrecktem Zeigefinger auf einen unsichtbaren Punkt in die Menge hinein. Die meisten Menschen haben den Rücken zur Kamera gewandt und stehen ruhig da oder laufen langsam umher. Sie blicken in die gleiche Richtung, in die auch der Finger zeigt. In der Ferne ist ein Stadtpanorama mit hellen Wohngebäuden am oberen rechten Bildrand zu sehen. Schnitt. In dieser Aufnahme ist die Kamera gegenüber den Menschen positioniert und blickt ihnen entgegen. Das Stadtpanorama ist nun weiter links im Rahmen positioniert und ist größer im Ausschnitt zu sehen. Die Menschen wenden sich um, während 
sie sich von einem Ansammlungspunkt links im Bildrahmen - manche schneller, manche langsamer - entfernen. Die stumme Aufnahme wackelt stark, schwenkt nach unten auf den Asphalt und zeigt nur noch die Beine der Menschen auf der Straße und den langen Schatten des Filmenden. Er hebt den rechten Arm mit seinem Kamerahandy in der Hand und filmt das Geschehen. Eine kleinere Gruppe von Menschen bewegt sich direkt auf ihn zu bis manche ganz im Bildausschnitt erscheinen. Sie scheinen jemanden in ihrer Mitte zu tragen. Der Filmende hebt auch den linken Arm und deutet auf diese Mitte. Die Gruppe bewegt sich an ihm vorbei und eine Person verdeckt dabei den Blick der Kamera kurzzeitig. Dann wird der Blick wieder frei und ein Mann, der ausgestreckt und mit leerem Gesichtsausdruck, leicht geöffneten Mund und regungslos auf dem Asphalt liegt, kommt ins Bild. Sein Körper liegt fast horizontal im Bildrahmen. Seine Beine und sein rechter Arm sind unnatürlich angewinkelt. Sein Hals, sein T-Shirt und seine linke Hand, die am ausgestreckten Arm auf dem Asphalt ruht, sind rot von Blut. Vom unteren Torso führt ein Streifen Blut auf dem Asphalt zu den blutigen Fingern der linken Hand. Die Aufnahme ist stark verwackelt. Ein dunkles Paar Beine läuft am oberen Bildrand vorbei. Am linken Bildrand tauchen nackte Zehen in Sandalen auf. Diese letzten Bilder dieser Aufnahme in Zeitlupe abspielend, ertönt Mohammeds Stimme in sanften arabischen Worten (mit deutschem Untertitel): „Der Zug von Deraa ist abgefahren. «Schnitt. Das blutverschmierte Gesicht des eben noch auf der Straße liegenden Demonstranten erscheint in ein weißes Leichentuch gewickelt seitlich auf einer Bahre liegend. Er fixiert den Zuschauenden mit leeren Augen. Hinter der Bahre stehen etwas abseits zwei Paar Beine. Über die stumme Aufnahme ist ein Pfeifton gelegt. Die Kamera fährt in einer Detailaufnahme langsam das Gesicht des Toten ab, zeigt die blutverschmierte Wange und das blutverschmierte Ohr und die verkrusteten Haare. Mohammed spricht mit sanfter Stimme weiter (mit deutschem Untertitel): »Mein Onkel half unserem Nachbarn.« Die Kamera schwenkt über Nase, Mund und Hals. Am unteren Bildrand wird eine Hand sichtbar, die den Kragen zur Seite schiebt, um den blutigen Hals für den Blick der Kamera freizumachen. "Sami hat Uhren in seinem Laden«, spricht Mohammed (mit deutschem Untertitel). Die Kamera entfernt sich ein kleines Stück, um das Gesicht des Toten voll im Bild zu zeigen. Im Hintergrund stehen weiterhin die zwei Paar Beine. Am Kopf des Toten bewegt sich ein dunkler Rumpf. Die Kamera fährt noch einmal über das Gesicht, um Wunden am Kinn und Hals einzufangen. Die Finger helfen wieder die Kleidung zur Seite zu schieben. »Ich erinnere mich an diese Leseübung«, sagt Mohammed aus dem Off (mit deutschem Untertitel). Die Hand schiebt das Leichentuch leicht zur Seite und hebt den Kragen an der anderen Seite des Halses an, um ein großes Einschussloch am Hals aufzudecken. Mohammed wiederholt (mit deutschem Untertitel): »Der Zug von Deraa ist abgefahren. «Die Kamera fixiert die klaffende, blutleere Wunde einen Moment lang in einer Detailaufnahme. »Als ich noch ein Kind war, mochte ich sie gern«, erläutert Mohammed abschließend (mit 
deutschem Untertitel). Dann entfernt sich die Kamera vom Toten. Die Hand zieht das Leichentuch über das Gesicht. Die Szene blendet langsam aus.

\subsection{EAU ARGENTÉE (2013) (F, R: Mohammed/Bedirxan): Le premier martyr (Kapitel 3.4.2)}

Auf die Titeleinstellung Le premier martyr (dtsch.: Der erste Märtyrer) in weißer arabischer und lateinischer Schrift auf schwarzem Hintergrund folgt die Szene einer friedlichen Protestmasse auf einem großen Platz in einer halbtotalen Einstellung. Das Geschehen wird in schlechter Videoqualität aus mitten der Protestmasse gefilmt. Die Menschen füllen einen langen Straßenzug, der von Wohngebäuden gesäumt wird. Viele halten ihre Hände oder Handykameras nach oben. Im Vordergrund ist ein grauer Sarg zu sehen, den die Menschen auf ihren Händen über ihren Köpfen mit sich tragen. Etwas weiter hinten sind rotweiße Tücher über den Köpfen zu sehen. Etwa in der Bildmitte hat sich ein Mann, der aus der Masse herausragt, umgewandt und blickt der Masse entgegen. Wegen der schlechten Bildqualität bleiben seine Gesichtszüge unklar. Das Licht ist gedämpft, der Himmel weiß von Wolken. Die Aufnahme ist stumm und verwackelt. Sie schwenkt unruhig nach rechts. Ein Wohngebäude kommt ins Bild, auf dessen Mauern ein Mensch steht und ein weiterer hockt. Der Stehende reckt seine Arme mit ausgestreckten Händen, die einen kleinen Gegenstand halten, nach vorne, als würde er die Menschenmasse mit seinem Handy filmen. Schnitt. Eine Totale eines blauen Himmels mit weißen Schleierwolken. In der unteren rechten Bildecke ist sehr klein ein dunkler, auf die Kamera zufliegender Hubschrauber am ansonsten leeren Himmel zu sehen. Die drehenden Rotorblätter sind zu erkennen. Schnitt. Weitere Bilder der Protestmasse. Im Synchronton sind nun die Sprechchöre zu hören, die mit »Öffne deine Pforten, Paradies! « in gelber Schrift am unteren Bildrand übersetzt werden. Der Sarg ist über die Masse gewandert und befindet sich nun weiter in der Bildmitte. Die Kamera zoomt heran. Eine weiße Aufschrift in arabischen Buchstaben wird auf dem Sarg sichtbar. Die dahinterliegende Protestmasse bleibt unscharf. Die Aufnahme wackelt stark. Den Bildvordergrund füllt jetzt eine Hand, die das Victory-Zeichen macht und der Kamera die Sicht versperrt. Schnitt. Ein anderer Teil der Straße gefüllt von Menschen, die sich langsam vorwärtsbewegen, ist zu sehen. Die Straße wird auf der rechten Seite von weiteren Menschen gesäumt, die den Zug von einer halbhohen Mauer aus an sich vorüberziehen lassen. Die Kamera filmt wieder aus der Mitte der Masse. Auch hier rufen die Menschen in Sprechchören auf Arabisch: »Revolution, Revolution! Gegen Unrecht, gegen Tyrannei! Revolution, Revolution! Gegen Unrecht, gegen Tyrannei! Es gibt keinen Gott außer Allah!« Manche Menschen halten auch hier Kamerahandys in der Hand und filmen das Geschehen. Die Aufnahme wackelt wieder stark. Einige Menschen wen- 
den ihren Kopf nach rechts und richten ihren Blick über die Mauer hinweg. Aus der Mitte der Masse taucht ein graues Auto auf, das sich rückwärts langsam durch die Masse zunächst auf die Kamera zu, dann links an ihr vorbei schiebt. Schnitt. Die Aufnahme zeigt in einem Ausschnitt von weiter rechts die Mauer am unteren Bildrand, die von vereinzelten Menschen überwunden wird. Dahinter ist eine weitere Menschenmenge zu sehen, die sich etwas verstreuter von der Kamera entfernt. Die Sprechchöre sind weiter zu hören. Schnitt. Der Hubschrauber am Himmel fliegt in einer halbtotalen Einstellung geradewegs auf die Kamera bzw. die Protestmasse zu, in deren Mitte sie sich befindet. Durch die Sprechchöre hindurch wird das Rotorengeräusch lauterwerdend hörbar. Schnitt. Wieder erscheint die Protestmasse vor der Mauer im Bild. Viele der Menschen drehen sich nun nach rechts, den Blick in Richtung Himmel gerichtet. Eine ganze Reihe von Menschen steht auch auf der Mauer, ihren Blick nach rechts gerichtet. Nur die Sprechchöre sind zu hören. Schnitt. Der Hubschrauber erscheint seitlich in einer halbnahen Einstellung. Er fliegt vom rechten Bildrand nach links. Nur die Rotoren sind laut zu hören. Die Kamera schwenkt mit ihm. Der Hubschrauber ist nun deutlich als militärisch zu erkennen. Schnitt. Ein anderer Ausschnitt der Protestmasse erscheint im Bild. Sie ruft (mit deutschem Untertitel): »Erhebe dich, Hauran!« Im Bildvordergrund rennt ein Mann im weißen Hemd plötzlich quer nach rechts aus der Masse heraus und verschwindet am rechten Bildrand. Schnitt. Der Hubschrauber fliegt in Zeitlupe in einer halbnahen Einstellung und in entgegengesetzter Flugrichtung von links nach rechts über die Kamera hinweg. Die Rotoren werden lauter. Schnitt. Es folgt ein Ausschnitt der Protestmasse in halbnaher Einstellung. Zuerst sind nur die Hinterköpfe und Rücken nah beieinanderstehender Menschen zu sehen. Dann schwenkt die Kamera nach oben und zeigt den ganzen Straßenzug mit der Protestmasse. Das Bild beginnt künstlich stark zu wackeln. Die Pixel werden größer. Die Sprechchöre werden mehr und mehr von Rotoren überlagert. Das Wackeln nimmt zu, bis das Bild aus dem Rahmen fällt. Schnitt. Rötlicher, in faltengeworfener Stoff ist zu sehen, dann der Straßenasphalt in groben Pixeln und schnellwechselnde Bilder in hauttönen, dunklen und hellen Schatten, Blut auf dem Asphalt und zu nahe Aufnahmen von bekleideten Körpern. Der Wind verfängt sich im Mikro und die Rotoren haben das gesamte Audio eingenommen. Dann ertönen Schreie und kreischende Stimmen vor wechselnden abgerissenen Bildern. Einzelne >Allah-Akbar<-Schreie sind zu hören. Immer öfter taucht der Asphalt, bedeckt mit großen Blutlachen, in diesen Bildern auf. »Bekenne dich! Sprich dein letztes Gebet, Imad!« ruft eine Männerstimme auf Arabisch (mit deutschem Untertitel). Am unteren Bildrand taucht eine Blutspur auf dem Asphalt und zuerst blutbedeckte Finger, dann ein blutverschmierter Haarschopf auf. Die Haare sind grau und kurz geschnitten. Die Einstellung bleibt auf dem blutigen Schopf vor den Blutlachen auf dem Asphalt fixiert. Kurzzeitig reißt das Bild ab und zeigt grauen, in faltengeworfenen Stoff, kommt dann aber zum blutigen Haarschopf zurück. Hinter den Schreien und Rufen ertö- 
nen dumpf die Rotorblätter. »Bekenne dich, Imad!«, ruft die Männerstimme immer und immer wieder (mit deutschem Untertitel). Am oberen Bildrand kommt kurzzeitig die Spitze eines Fußes mit offenen Sandalen ins Blickfeld, der auf dem Asphalt in unmittelbarer Nähe zum blutigen Schopf steht. Die Männerstimme beginnt unerträglich zu kreischen. Weitere schreiende Stimmen kommen hinzu. Der Ton bricht ab. Das Bild läuft noch einen kurzen Moment weiter, zeigt den blutigen Schopf, dann den Rücken des Mannes und fremde Arme, die ihn halten. Schnitt.

\subsection{EAU ARgentÉE (2013) (F, R: Mohammed/Bedirxan): Mohammeds gestohlene Kamera (Kapitel 3.4.2)}

Durch einen Metallzaun im Kupferton hindurch wird in einer halbtotalen Einstellung eine Menschenmasse gefilmt, die flüchtend eine hinter dem Zaun liegende Straße entlangrennt. Die Aufnahme ist unscharf und verpixelt. Alles übertönende Schüsse unsichtbarer Waffen sind zu hören. Die Kamera bewegt sich in kurzen Schwenks mal etwas nach rechts und mal etwas links. Vereinzelte Autos, eines sogar im Rückwärtsgang, und ein Motorrad rasen schnell die Straße entlang in die gleiche Richtung wie die fliehenden Menschen. Einige der Menschen suchen auf der Straße hinter einer baulichen Fahrbahntrennung Schutz. Der Strom aus Menschen reißt ab. Ein einzelner Mann in dunkler Kleidung rennt stolpernd über die Straße. Mohammeds Stimme spricht auf Arabisch aus dem Off (mit deutschem Untertitel): »Heute Morgen...«. Schnitt. Die Kamera nimmt das Geschehen jetzt von etwas weiter rechts auf. Eine Strebe des Zauns verläuft in der linken Bildhälfte vertikal durch das Bild. Der Synchronton ist verstummt. Die Menschen fliehen in eine Stichstraße. Eine große Pfütze füllt den Asphalt. Vor und in der Pfütze liegen Körper von Getroffenen. Einzelne Menschen rennen an ihnen vorüber und durch die Pfütze. Jeder Tritt, der die Pfütze trifft, wird aus dem Off mit einem lauten Plätschern begleitet. Die Kamera bewegt sich in einem unruhigen Schwenk nach links, fängt den kupferfarbenen Zaun und eine Hauswand mit davor platzierten Tonkrügen ein. »...nahm mir jemand die Kamera«, spricht Mohammed im Off weiter (mit deutschem Untertitel). Vor schattigen, verpixelten Bildern der Häuserwand, die zwischendurch von schwarzen Einstellungen unterbrochen werden, hört man das schwere Atmen eines Mannes. „Und das Kino begann...", spricht Mohammed (mit deutschem Untertitel). Vor den gleichbleibenden Bildern erzählt er weiter (mit deutschem Untertitel): »Ich folgte demjenigen, um sie mir zurückzuholen.« Das Bild wechselt zu einer nahen Aufnahme eines Straßenpflasters. Laufende Schritte, aber keine weiteren Umgebungsgeräusche sind zu hören. Es ist unklar, ob es sich dabei um den Synchronton der Aufnahme oder ein später hinzugefügtes Audio handelt. Am oberen Bildrand läuft ein Paar Beine in weißen Turnschuhen durch das Bild. Die Kameraführung ist sehr unruhig und erweckt den Eindruck, als wür- 
de der Filmende mit der Kamera in der Hand laufen. Die Kamera schwenkt leicht nach oben, um mehr von der Straße einzufangen. Die Straße ist nass. Rechts und links sind die abgeschnittenen Körper laufender Personen zu sehen, die sich geradeaus auf eine Straßenkreuzung zubewegen. Gemeinsam mit den Schritten ist nun auch ein angestrengtes Atmen zu hören. Die Kamera bewegt sich mit den Schritten auf zwei Menschen zu, die ausgestreckt auf dem Asphalt liegen. Blut säumt ihre Körper. Von allen Seiten eilen Menschen zu ihnen. Die Kamera bleibt vor einem der Körper stehen. Ein Mann, bekleidet mit einer Jeans und weißen Turnschuhen, liegt auf dem Rücken auf der Straße. Sein Gesicht ist blutüberströmt. Seine Mütze ist vom Kopf gefallen und liegt wenige Zentimeter von seinem Kopf entfernt in einer großen Blutlache. Zwei Männer greifen den Getroffenen an den Armen. Die Kamera bewegt sich nach links. Drei weitere Männer liegen getroffen auf der Straße. Der Mann am nächsten zur Kamera hebt auf dem Rücken liegend seinen Kopf. Ein Mann greift nach seinem Arm und versucht ihm aufzuhelfen. Die Bilder sind stumm. In einer verwackelten Bewegung fährt die Kamera um die Körper herum nach rechts auf den zweiten Getroffenen zu und entfernt sich dann wieder von den Körpern. Die Kamera fährt nach links und fängt die Blutlache des ersten Getroffenen ein. Seine Mütze liegt immer noch dort, aber sein Körper wurde fortgetragen. Die Kamera folgt der Blutspur. Ein verlorener Schuh liegt auf dem Asphalt. Laufende Schritte ertönen. Ein Stück die Straße hinauf findet die Kamera den Körper des ersten Getroffenen wieder, der an den weißen Turnschuhen erkennbar ist, die zur Kamera gerichtet sind. Mehrere Männer stehen um ihn herum. Die Schritte verstummen. Die Kamera bewegt sich um den Getroffenen herum, während die Männer ihn an Armen und Beinen packen und gemeinsam forttragen. Die Kamera filmt das Geschehen nun von Vorne. Der blutüberströmte Kopf des Getroffenen ist zur Kamera gerichtet. Blut tropft auf die Straße. Die Kamera harrt einen Moment aus, während der Getroffene aus dem Bild getragen wird. Dann schwenkt sie nach rechts, um einen weiteren Getroffenen einzufangen. Sein rechtes Bein wurde angeschossen. Rote Blutströme zeichnen sich auf dem grauen Stoff seiner Hose ab. Er liegt auf der Seite und bewegt seine Beine weiter, als würde er noch immer vor den Schüssen davonlaufen. »Ich bemerkte, dass ich ihm Anweisungen gab«, spricht Mohammed über die ansonsten tonlosen Bilder (mit deutschem Untertitel). Die Kamera schwenkt schnell nach links. Auf der Straße stehende Personen kommen kurzzeitig abgeschnitten ins Bild, bevor die Kamera in einer Detailaufnahme nackte Füße einfängt, an denen Blut herunterläuft. »Beweg die Kamera nicht!«, ruft Mohammed (mit deutschem Untertitel). Die dazugehörigen Beine, in Jeans gekleidet und mit Blut getränkt, füllen das Bild. »Statische Einstellung!« ergänzt Mohammed (mit deutschem Untertitel). Die Beine werden angehoben von Helfenden, von denen nur abgeschnittene Arme oder Beine im Bild zu sehen sind. Schnitt: Szenenwechsel. Der blutige Schopf des grauhaarigen Mannes vor dem Straßenasphalt mit Blutströmen aus der Le-premier-martyr-Sequenz erscheint im 
Bild. Das Bild wackelt und friert dann bei einer Aufnahme der Blutströme ein, vor denen die Fußspitze in offenen Sandalen und die nackten Zehen zur Kamera gerichtet auf dem Asphalt steht. Schnitt: Szenenwechsel. Eine weitere Straßenszene von Getroffenen und Helfenden. Ein Mann wird an Armen und Beinen durch das Bild getragen. Es ist kein Blut zu sehen. Dahinter liegt eine weitere Person auf der Straße schräg vor einem weißen Lastwagen, der mitten auf der Straße steht. Der obere und vordere Teil des Lastwagens sind vom Bildrahmen abgeschnitten. Viele Menschen stehen still auf der Straße. Auch diese Aufnahme ist ohne Ton. »Eine statische Einstellung ist schön.«sagt Mohammed im Off (mit deutschem Untertitel). Schnitt. Der Getroffene vor dem weißen Lastwagen ist in einer Totalen, auf dem Rücken und mit ausgestreckten Armen auf der Straße liegend zu sehen. Die Kamera schwenkt nach links, fängt ein laufendes Paar Beine vor dem Lastwagen ein und schwenkt dann wieder nach rechts zurück zu dem Getroffenen. Auch diese Bilder sind stumm. Ein neben ihm stehender Mann hat sich vorgebeugt und seine linke Hand auf die Brust des Getroffenen gelegt, als ob er fühlen wollte, ob er atmet und noch lebt. Das Bild ruckelt. Schnitt. Das Gesicht des Getroffenen in einer Großaufnahme. Von der Augenpartie an ist es blutüberströmt. Augen und Mund sind leicht geöffnet. »Der Filmregisseur war tot«, sagt Mohammeds milde Stimme (mit deutschem Untertitel). Der Mund bewegt sich leicht in einer nach Luft schnappenden Bewegung. Dann bleibt das Gesicht regungslos. Eine Hand kommt von links ins Bild und legt sich vorsichtig auf die Wange des scheinbar gerade Gestorbenen. Ein sanftes Summen ertönt. Das Bild blendet schwarz aus.

\subsection{EAU ARgentÉE (2013) (F, R: Mohammed/Bedirxan): Ciné-Club (Kapitel 3.4.2)}

Über die Titeleinstellung Ciné-Club (dtsch.: Filmklub) in weißer arabischer und lateinischer Schrift auf schwarzem Hintergrund ist das Abspielgeräusch einer Filmrolle gelegt. Es folgt ein stummes Video einer belebten Straßenszene in geringer Aufnahmequalität. Im Vordergrund liegen mehrere Männer mit auf dem Rücken verbundenen Händen bäuchlings und ungeordnet auf dem Asphalt. Teilweise liegen sie aufeinander. Zwei Männer mit militärischen Westen stehen inmitten der Liegenden. Die Kamera filmt aus ihrer Höhe. Der Mann am rechten Bildrand tritt einem Am-Boden-liegenden mit seinem schweren Stiefel mittig auf den Rücken und wendet sein Gesicht dabei zur Kamera. Mit einem Schnitt ist der Mann mit Stiefel verschwunden. Vom rechten Bildrand taucht ein anderer Mann mit einem Schlagstock in der rechten Hand auf und schlägt den gleichen Am-Boden-liegenden damit kräftig auf den Rücken, während er mit der linken Hand ein Handy an sein linkes Ohr hält. In der linken Armbeuge hält er ein Maschinengewehr ungelenk an sich gedrückt. »Realtisches Kino«, erscheint in gelber Schrift am unteren Bild- 
rand, während Mohammeds sanfte Stimme im Off dieselben Worte auf Arabisch spricht. Der Am-Boden-liegende dreht sich langsam auf den Rücken und wird von dem Mann zu seiner Linken ins Gesicht getreten. Das Bild schwankt unruhig hin und her. Der Mann zur Linken tritt erneut zu, während sich der Am-Boden-liegende $\mathrm{zu}$ einer sitzenden Position aufrichtet. Von rechts folgt ein weiterer Hieb mit dem Schlagstock auf seinen Rücken. Im Hintergrund ist $\mathrm{zu}$ sehen, wie weitere Männer Am-Boden-liegende schubsen und an ihnen zerren. Unter weiteren Hieben mit dem Schlagstock richtet sich der Mann auf und läuft mit schnellen Schritten die Straße hinauf. Schnitt. In einer halbtotalen Einstellung blicken wir eine sonnenerleuchtete Straße hinunter, auf der Männer mit nacktem oder leicht bekleidetem Oberkörper verstreut und rücklings liegen oder sitzen. Ihr Blick ist von der Kamera abgewandt. Sie schauen die Straße hinunter oder in den Himmel. Manche strecken ihre Arme nach oben und machen das Victory-Zeichen. Die Aufnahmequalität ist schlecht. Die Straße ist umgeben von grünen Böschungen, Strommasten und entfernten hohen Schornsteinen. Die Kamera zoomt heran und fängt hinter einem hockenden Mann mit nach oben ausgestreckten Armen einen sich nähernden Panzer ein. Manche Männer stehen auf und entfernen sich von dem herannahenden Panzer aus dem Bild. Dabei wird die Sicht der Kamera kurzzeitig versperrt. »Bizarres Kino«, erscheint in gelber Schrift am unteren Bildrand, während Mohammeds sanfte Stimme im Off dieselben Worte auf Arabisch spricht. Das Bild wackelt unruhig hin und her. Der Sound der Filmrolle wird durch ein lauterwerdendes Rauschen übertönt, das der Synchronton des heranrollenden Panzers sein könnte. Manche Männer geben ihre Position auf der Straße nicht auf und blicken mit nach links gewendetem Kopf dem Panzer entgegen. Der Synchronton verstummt wieder. Die Filmrolle ist im Off wieder zu hören. Schnitt. In einer halbnahen Einstellung hockt ein gefesselter Mann mit über dem Kopf gezogenem, weißem T-Shirt rücklings vor einer hellgrauen Wand. Sein Oberkörper ist nach Vorne gebeugt. Sein T-Shirt ist über sein Gesicht gezogen, das nach unten zum Boden gewandt ist. Hinter ihm ragt das Ende eines Kabelbinders in die Höhe. Am rechten Bildrand steht ein Mann in militärischer Kleidung seitlich neben ihm. Sein Kopf ist $\mathrm{ab}$ den Schultern vom oberen Bildrand abgeschnitten. In der rechten, $\mathrm{zu}$ dem Hockenden gerichteten Hand hält er eine Handfeuerwaffe. Die linke, zur Kamera gerichtete Hand steckt locker in seiner Hosentasche. »Kino des Mörders«, erscheint in gelber Schrift am unteren Bildrand, während Mohammeds sanfte Stimme im Off dieselben Worte auf Arabisch spricht. In Zeitlupe hebt der Mann die Handfeuerwaffe an und richtet sie im schrägen, nach unten gerichtetem Winkel auf den Kopf des Hockenden. Die Kamera zoomt langsam an den Hockenden heran, während ein lauter, hallender Schuss ertönt und ein Lichtfleck an der Öffnung der Waffe aufleuchtet. Der Hockende zuckt zusammen und kippt langsam nach hinten. Die Aufnahme fährt im normalen Tempo fort. Der Mann senkt die Waffe und entfernt sich mit schnellen Schritten von dem Hockenden, auf dessen Hinterkopf und wei- 
ßem T-Shirt im Brustbereich Blut zu sehen ist. Die Aufnahme wechselt zu einem hellgrauen, flackernden Bild. Schnitt. Eine alte zahnlose Frau im dunkeln Tschador erscheint in einer Großaufnahme. Im Synchronton ist ihr angestrengtes, erschöpftes Atmen zu hören. Sie lehnt ihren Kopf im schrägen Winkel links gegen eine graue Wand. Hinter ihr ist im schrägen Winkel eine Eingangstür zu sehen. Große Schatten füllen das Bild. »Kino des Opfers«, erscheint in gelber Schrift am unteren Bildrand, während Mohammeds sanfte Stimme im Off dieselben Worte auf Arabisch spricht. Die Frau blickt rechts und links mit weit aufgerissenen Augen und erschrocken geöffnetem Mund an der Kamera vorbei. Dann presst sie die Lippen fest zusammen, blinzelt mehrmals und blickt schließlich betroffen in die Kamera. Wieder setzt das Abspielgeräusch der Filmrolle ein. Schnitt. Eine großverpixelte und in den Rotbereich verschobene Aufnahme eines Neugeborenen, leicht bedeckt mit einem weißen Tuch, erscheint. Die Aufnahme ist stumm und wird nur von dem Abspielgeräusch der Filmrolle begleitet. Das Neugeborene liegt auf der linken Seite. Vom rechten und oberen Bildrand aus durchtrennen zwei Hände mit einer chirurgischen Schere seine Nabelschnur. Vom linken Bildrand legt sich eine Hand schützend auf den Kopf des Neugeborenen. Schnitt. Eine unterbelichtete Szene eines Trauerzuges in einer halbtotalen Einstellung aus der Vogelperspektive. In der Bildmitte, fast parallel zur Bildhorizontalen ist ein offener, schwarzer Sarg, ausgekleidet mit weißem Stoff, zu sehen. Darin liegt ein Toter, der in ein weißes Tuch gehüllt ist. Weißgraue Papierfetzen regnen auf die dunkelgekleideten Menschen herab. Die stummen Bilder werden wieder nur von dem Abspielgeräusch der Filmrolle begleitet. »Poetisches Kino«, erscheint in gelber Schrift am unteren Bildrand, während Mohammeds sanfte Stimme im Off dieselben Worte auf Arabisch spricht.

\subsection{EAU ARGENTÉE (2013) (F, R: Mohammed/Bedirxan): Die Geschichte ist... (Kapitel 3.4.2)}

Bedirxan geht filmend durch die Ruinen der Stadt Homs. Sie filmt mit der Kamera auf Bodenhöhe die schutterfüllten Straßen, tote Äste und eine große Pfütze. Über die Aufnahme sind ein Plätschern und ein leiser, hoher Pfeifton gelegt. »Havalo...«, spricht Bedirxan aus dem Off (mit deutschem Untertitel). Schnitt. Eine ramponierte Matratze und ein kleines Plastikpferd liegen im Schutt. Plätschern und Pfeifen sind verstummt. Bedirxans Schritte auf körnigem Untergrund sind im Synchronton zu hören. »...magst du Romane? «, fährt Bedirxan im Off fort (mit deutschem Untertitel). Die Kamera schwenkt linksherum. Bedirxans Schatten legt sich kurz über die Matratze und den Schutt. Sie läuft mit zielstrebigen Schritten vorwärts, während die Kamera den schutt- und müllbedeckten Boden einfängt. »Magst du Geschichten?«, fragt sie im Off (mit deutschem Untertitel) und bleibt vor einer langen verlassenen Häusergasse stehen. Keine Menschen und keine Tiere sind zu se- 
hen. Schnitt. Inmitten der grauen Einöde städtischer Trümmer liegt ein roter Plastikkanister. An der rechten Häuserzeile, auf einem Garagentor und zwischen zwei Türöffnungen steht in schwarzer, arabischer Schrift groß etwas geschrieben. Auch an der Häuserzeile gegenüber der Kamera sind arabische Aufschriften in schwarzer Farbe zu sehen. »Schläfst du?«, fragt Bedirxan weiter (mit deutschem Untertitel). Mit langsamen, kaum hörbaren Schritten geht sie auf den Kanister zu. Die Kamera liegt nun höher, filmt etwa aus Bauchhöhe die Straße hinunter und bewegt sich an dem Kanister vorbei langsam um eine Hausecke. »Die Geschichte ist, dass ich laufe und filme«, spricht Bedirxan (mit deutschem Untertitel). Schnitt.

\subsection{2 squidd81 (2016): ISIS Insurgent films own death on GoPro - Graphic Footage (Kapitel 3.5)}

Das Video beginnt mit einer Nahaufnahme von braunen Sträuchern, in deren Mitte Trümmerstücke liegen. In der linken oberen Bildecke ist ein weißes Rechteck über das Bild gelegt, das sich das ganze Video lang dort befindet. Die Kamera schwenkt ruckartig in einer diagonalen Linie nach oben zum wolkenverhangenen Himmel. Die Lichtverhältnisse sind entsprechend der Bewölkung leicht gedämpft. Zwei Stimmen - eine aus etwas weiterer Entfernung, eine in unmittelbarer Nähe zur Kamera - rufen etwas auf Arabisch. Im unteren Teil des Bildes ragt ein Strommast in den Himmel. In der unteren linken Bildecke sind entfernte Gebäudeelemente in einem leicht nach links geneigtem Winkel zu erkennen. Die Sicht der Kamera wird durch Regentropfen auf der Linse erschwert. Im Hintergrund sind entfernte Schüsse, ein stetes Motorengeräusch und in direkter Nähe zur Kamera das Laden einer Waffe zu hören. Der Wind verfängt sich im Mikro. Das Bild erschüttert, als ein lauter Schuss abgefeuert wird. Durch die rechte Bildhälfte bewegt sich ein kleiner, dunkler Gegenstand in einer Flugkurve. Zwei weitere Schüsse und kurze Erschütterungen des Bildes folgen kurz aufeinander. Das Bild schwankt dabei leicht hin und her. Entfernt ertönt eine Stimme. Ein erneuter Schuss mit einem davonfliegenden Gegenstand. Das Bild kippt nach links als würde der Filmende mit der Kamera umfallen. Ein großer Strauch und ein Mann in dunkler Kleidung kommen in mittelbarer Nähe des Filmenden kurz ins Bild, während eine weitere Stimme in direkterer Nähe zur Kamera etwas ruft. Schnell richtet sich die Kamera wieder nach rechts, waagerecht zur Horizontlinie aus. Das Bild schwenkt dabei über die braunen Sträucher mit Trümmerstücken und stoppt kurzzeitig bei dem Blick auf den Strommasten und das entfernte Gebäude. Ein schneller Schwenk nach links folgt, der an eine Kopfbewegung erinnert. Die Kamera bewegt sich in einer gehenden Bewegung auf den Mann zu, der jetzt als militärisch ausgerüsteter Kämpfer, mittleren Alters mit schulterlangen, dunklen Haaren erkennbar ist. Auf dem Rücken trägt er einen Rucksack und wirft sich gerade den Gurt eines 
Gewehrs über die linke Schulter. Direkt gegenüber dem Mann bleibt die Kamera stehen und schwenkt schnell nach rechts, zurück zu dem Strommasten und dem Gebäude. Die Kamera bewegt sich wenige Schritte nach Vorne und sackt etwas $\mathrm{ab}$ als würde sich der Filmende hinhocken. Auf der Linse befinden sich weiterhin mehrere Wassertropfen, die die Sicht leicht verstellen. Ein klackendes Metallgeräusch übertönt kurz die anhaltenden Schüsse und Stimmen im Hintergrund. Ein längliches Objekt, in dunklen Farbtönen und mit einer hellen Kappe kommt von unten schräg ins Bild, während die Kamera in ihrer Position verharrt und in leicht schwankenden Bildern die unmittelbare Umgebung von vertrockneten Sträuchern, Trümmerstücken und Stofffetzen einfängt. Einzelne laute Schüsse sowie das Positionieren und Laden von Gewehren sind zu hören. Die Kamera schwenkt wieder nach oben in den wolkenverhangenen Himmel und verweilt dort einige Sekunden. Im Hintergrund ist das Motorengeräusch nun wieder lauter $\mathrm{zu}$ hören. Das Bild wackelt in kurzen Bewegungen von links nach rechts, von rechts nach links. Nur noch vereinzelte, entfernte Schüsse fallen. Eine lautere männliche Stimme sagt etwas auf Arabisch. Aus der Entfernung setzt ein Schussfeuer ein. Ein dumpfes Aufschlaggeräusch in unmittelbarer Nähe zur Kamera ertönt und das Bild kippt nach links zu Boden. Es fängt die braunen Sträucher mit Trümmerstücken ein, prallt noch einmal kurz nach oben und bleibt dann statisch. Wieder folgt ein dumpfer Aufschlag. Mit einem Schmerzensaufschrei fällt die Kamera ganz zu Boden. Brauner Erdboden mit vertrockneten, einzelnen Grashalmen und Blättern erfüllen das Bild im Vordergrund. Die Horizontlinie ist fast senkrecht gekippt. Am rechten Rand durchschneidet ein schwarzes längliches Objekt senkrecht das Bild. Direkt dahinter, in der oberen rechten Bildecke, liegt ein Trümmerstein aus Beton. Das Mikrofon der Kamera gibt laute Geräusche wieder, die unbeabsichtigte Berührungen suggerieren. Im Hintergrund sind Ausrufe zu hören. Ein lautes, schiebendes Geräusch ertönt, während sich die Kamera nach vorne bewegt und sich im Bildvordergrund Erde vor der Linse auftürmt. Wieder ertönen Schüsse und Rufe. Mit einem dumpfen Aufschlag erschüttert die Kamera kurz. Die Kamera bewegt sich leicht und kippt minimal weiter nach links. Hohle Bewegungsgeräusche am Mikro ertönen. Auf dem Boden liegend macht die Kamera einen Satz nach links und verweilt in einer fast senkrechten Horizontlinie bei einem anderen Teil der braunen Sträucher mit Trümmerstücken. Dahinter geht eine Person in Deckung. Das Bild verweilt für knapp 30 Sekunden in dieser Einstellung. Mit nur kleinen Bewegungen der Kamera verschiebt sich das Bild nach rechts oder links, während schabende Geräusche und ein leises, angestrengtes Atmen zu hören sind, als würde der Filmende versuchen sich über den Erdboden zu ziehen. Dann rotiert das Bild um die eigene Achse nach links. In der unteren, rechten Ecke schwebt eine schemenhafte Hand durch das Bild. Das Bild steht fast auf dem Kopf, bevor es wieder zurückfällt und nun ein ganzer Unterarm im unteren rechten Viertel des Bildes auf den Boden fällt. Immer noch liegen einzelne Wassertropfen auf der Linse. Das angestrengte 
Atmen, das Schussfeuer im Hintergrund und das Motorengeräusch halten an. Einzelne Erschütterungen von ruckartigen Bewegungen der Kamera erfassen Bild und Ton. Nach ca. 20 weiteren Sekunden rotiert das Bild wieder um die eigene Achse nach rechts, bis es fast auf dem Kopf steht. Dann fällt es wieder leicht zurück und bleibt in einem schrägen Winkel über 180 Grad stehen. Erschöpfte Schmerzensrufe erfüllen das Audio. Sie nehmen den Rhythmus eines stoßartigen Atmens an. Die schmerzerfüllten Atemstöße werden nach und nach leiser und länger. Immer wieder sind Schüsse im Hintergrund zu hören. Dann folgt eine plötzliche Linksrotation des Bildes gemeinsam mit Bewegungsgeräuschen der Kamera. Das Bild bleibt kurzzeitig in waagerechter Horizontlinie stehen, wackelt leicht hin und her und fällt wieder in einer Linksrotation zurück mit senkrechter Horizontlinie. Die Einschläge der Schüsse werden stärker. Die schmerzverzerrten Atemstöße halten an. Mit einem über dem Erdboden schabenden Geräusch bewegt sich die Kamera nach hinten und zeigt nun wieder den Strommasten vor grauem Himmel in einem schrägen Winkel über $180 \mathrm{Grad}$. Vom unteren Bildrand fällt eine Hand senkrecht auf den Boden. Die Einstellung verweilt in der Großaufnahme der fast regungslosen Hand. Einzelne Finger zucken gemeinsam mit angestrengten Atemstößen vor anhaltenden Schüssen. Das Video endet unvermittelt.

\subsection{SaraNews (2015): 18 Syrian sniper killed precise shot to the head of the terrorist FSA (Kapitel 4.1)}

In einer halbtotalen Einstellung versuchen fünf Männer in ziviler Kleidung mit einem langen Stab, der an einem Ende zu einem Haken geformt ist, abwechselnd etwas um eine Hausecke herumzuziehen. Umgebung und Lichtverhältnisse suggerieren, dass das Geschehen draußen bei Tag in einer ländlichen Wohnsiedlung stattfindet. Die Männer werden von hinten gefilmt. Der Boden ist erdig sandig und mit grauen Steinen gesäumt, hinter denen struppige, gelbe Grasbüschel stehen. Im Bildhintergrund steht ein kleiner grüner Baum neben einer Mauer aus großen rotbraunen Steinen. Die Kamera ist leicht nach links gekippt. In direkter Nähe zur Kamera ertönt eine laute Männerstimme, die zu den fünf Männern an der Hausecke spricht. Im Hintergrund sind weitere männliche Stimmen und entfernte Schüsse zu hören. Die Männer wirken unruhig, während einer von ihnen mit dem Stab an der Hausecke hantiert und zu ziehen beginnt. Einige von ihnen tragen schusssichere Westen, Maschinengewehre oder anderes militärisches Equipment bei sich. Einer der Männer entfernt sich aus dem Blickfeld der Kamera, die auf die übrigen vier Männer fixiert bleibt und langsam heranzoomt. Wieder ertönen laute Schüsse und nun auch Granateneinschläge im Hintergrund. Ein weiterer Mann im Bildausschnitt ist nur sein Oberkörper sichtbar - erscheint kurz im Bild. Eine aufgeregte Stimme ertönt. Andere Stimmen reihen sich ein. Einer der Männer 
nimmt ein Maschinengewehr, hält es hoch und um die Hausecke herum, während der Stab an einen anderen Mann weitergereicht wird. Die Kamera schwenkt kurz nach links. Als sie die Hausecke wieder fokussiert, entfernen sich die Männer nacheinander von der Hausecke. Der Mann mit dem Gewehr hat seine Waffe gesenkt und verweilt mit dem Rücken gegen die Wand an der Hausecke gelehnt. Laute Ausrufe ertönen. Der Mann mit Gewehr gestikuliert mit der rechten Hand an einem unsichtbaren Gegenüber im linken Off. Die Kamera schwenkt leicht nach links und dann wieder zurück zur Hausecke. Das Bild ist leicht nach rechts gekippt, während sich im Hintergrund die Männer lautstark etwas zu rufen. Nun entfernt sich auch der Mann mit dem Gewehr etwas von der Hausecke und die Kamera zoomt näher an diese heran. Wieder ertönen laute Schüsse. Hinter der Hausecke wird ein im Schutt auf der Seite liegender Oberkörper sichtbar. Völlig regungslos liegt eine Hand auf dem Gurt eines Maschinengewehrs. Die Kamera wackelt hin und her und zoomt schließlich wieder heraus, um einen größeren Ausschnitt der Hausecke einzufangen. Von rechts nähert sich wieder einer der Männer mit dem langen gebogenen Metallstab der Hausecke, kniet sich auf eine davor drapierte Polsterunterlage und versucht wieder den regungslosen Körper um die Hausecke zu ziehen. Die Kamera filmt nun in Bodenhöhe in einer halbnahen Einstellung. Drei Männern gelingt es mit vereinten Kräften schließlich, den Körper um die Ecke zu befördern. Als ein Gesicht der Männer im Bild erscheint, ist es stark verpixelt. Aufgeregtes Reden und Schüsse sind zu hören. Einer der Männer betrachtet die Schusswunde am Kopf des Getroffenen, der ausgestreckt auf dem Rücken liegt und dessen Gesicht rot von Blut ist. Die Kamera ist unruhig: zoomt mal etwas an die Szene heran, dann wieder heraus. Zwei Männer überprüfen die Vitalzeichen des Getroffenen, während im Hintergrund ein weiterer Mann versucht auch noch das Maschinengewehr mit dem langen Stab um die Hausecke herum zu befördern. Die übrigen Männer stehen um den Getroffenen und unterhalten sich, wobei ihre Gesten annehmen lassen, dass es dabei um den Getroffenen geht. Als die Überprüfung der Vitalzeichen abgeschlossen ist, bringt einer der Männer eine große rote Decke mit floralem Muster, die auf dem Boden ausgebreitet wird. Der Filmende geht wenige Schritte zurück, bis die Szene in einer halbtotalen Einstellung zu sehen ist. Als auch das Maschinengewehr geborgen ist, geht dieser Mann hinüber zum Getroffenen und durchsucht mit einem weiteren Mann die militärische Einsatzweste des Getroffenen. Ein anderer bricht in Wehklagen aus, das aus der ohnehin geräuschvollen Kulisse hervorklingt. Die Kamera schwenkt zu ihm nach rechts als er sich etwas entfernt von dem Getroffenen an der Hauswand abstützt, zoomt bis zur halbnahen Einstellung heran und zeigt kurz sein Wehklagen. Es sind keine weiteren Schüsse zu hören. Die Kamera schwenkt wieder nach links, zoomt etwas heraus und zeigt die übrigen Männer, wie sie den Getroffenen weiter durchsuchen. Noch weitere Männer stoßen hinzu, so dass nun mehr als zehn Personen an der Szene beteiligt sind und einen losen Kreis um den Getroffenen bilden. Einer der 
Männer spricht in ein Funkgerät. Wieder fokussiert die Kamera den Wehklagenden, der sich in seinem deutlich hörbaren Schmerz von der Szene abwendet. Mit einem Linksschwenk richtet sich die Kamera zurück auf das Geschehen um den Getroffen: drei der Männer beginnen den Getroffenen zu entkleiden, während die anderen dieses Geschehen beobachten. Die Aufnahme endet unvermittelt.

\subsection{SNN Shaam English News (2013a): SNN I Syria I Damascus Rural I Soldier's Grief Over Dead Brother I Jun 17, 2013 (Kapitel 4.1.1)}

Das Video setzt mit einer Großaufnahme des leblosen Gesichts eines Mannes mittleren Alters ein. Seine dunklen Haare und sein Bart sind kurz geschnitten. Um seinen Kopf, der auf einem weißen Tuch ruht, ist ein schmaler Streifen eines weißen Verbandes gebunden. Im Hintergrund sind männliche Stimmen zu hören. Das Licht ist gedämpft und scheint von einer künstlichen Lichtquelle über dem Toten $\mathrm{zu}$ kommen. Im oberen linken Bildrand ist ein wackelndes goldenes Logo mit arabischen Schriftzeichen zu sehen, das links vom Bildrahmen abgeschnitten ist. Darunter ist in weißer lateinischer Schrift eine Einblendung zu lesen, die nach wenigen Sekunden verschwindet: »[Logo: Addumair]«. In direkter Nähe zur Kamera spricht ein Mann etwas, das am unteren Bildrand in einer Einblendung in weißer Schrift übersetzt wird: »Cameraman: Casuality, fighter and soldier Mahdi Shahib.« Das Bild zoomt langsam heraus bis zur halbnahen Einstellung, während der Mann weiterspricht: »He was killed June 15, 2013 and was from the city of Addumair. We pray that God accepts him. « Der Tote trägt eine khakifarbene Jacke im Pilotenstil. Seine Hände ruhen unterhalb seiner Brust und sind mit einem Verband zusammengebunden. Überblende. Die Kamera ist seitlich neben dem Toten etwa auf Hüfthöhe positioniert und filmt in einer halbnahen Einstellung wie ein Mann mit lockigem Haar sich über den Toten beugt, seinen Kopf zwischen Hals und Schulter des Toten versenkt und ihn umarmt. Er schluchzt. Die Kamera fährt etwas näher an den Trauernden heran. Im Hintergrund sind weiterhin männliche Stimmen zu hören. Auf dem Rücken trägt er einen militärisch wirkenden Rucksack, an dem ein Raketenwerfer befestigt ist. Eine Stimme aus dem Hintergrund wird am unteren Bildrand übersetzt: »Man: That's enough, that's enough. Just pray for your brother that God accepts him. «Überblende. Der schluchzende Mann sitzt nun auf einer Bahre. Die Kamera filmt von einer Position ihm Gegenüber leicht links in einer halbnahen Einstellung. Drei Männer sitzen oder knien trostspendend um den Trauernden herum. Im rechten Bildhintergrund steht ein Rollstuhl. Daneben stehen hohe Pappkartons. Der schluchzende Trauernde hat den Oberkörper nach Vorne gebeugt und hält seine Hände vors Gesicht. Im Hintergrund sind weiterhin Stimmen zu hören. Am unteren Bildrand liest sich:»Man2: Is there anyone else?« Lauter im Hintergrund ertönt eine Stimme über einen Lautsprecher. Die 
Kamera zoomt langsam an den Trauernden heran bis zur Nahaufnahme. Er hält sich ein Taschentuch an die Augen und schluchzt ununterbrochen weiter. Am unteren Bildrand liest sich weiter: »Radio: Saeed. Saeed. Muhammad Saeed. Man2: God is our source of strength and we have relied solely on Him. Radio: Saeed. Saeed. Muhammad Saeed. Saeed. Saeed. Muhammad Saeed.« Der Mann rechts von ihm erhebt sich am linken Bildrand und geht rechts an der Kamera vorbei aus dem Bildrahmen heraus. Der Mann rechts neben dem Trauernden, von dem nur noch der Oberkörper im Bildausschnitt erscheint, streicht sanft mit der rechten Hand über seinen Rücken. Der Mann vor dem Trauernden hat seinen rechten Arm auf sein Knie gestützt und stützt damit seinen Kopf mit einem ratlosen Gesichtsausdruck. Während die Kamera heranzoomt, verschwindet letzterer aus dem Bildausschnitt. Als die Kamera wieder langsam herauszoomt hat auch er sich von dem Trauernden entfernt. Der Mann links neben dem Trauernden lässt seine Hand weiter auf dessen Rücken ruhen und blickt ebenfalls ratlos in das rechte Off. Eine männliche Stimme ertönt im Hintergrund. Am unteren Bildrand liest sich: »Man3: Son, praise God. Praise God! He is chosen by God.« Der Mann neben dem Trauernden wendet seinen Kopf zu ihm hinunter. Mit einer seufzenden Bewegung wendet er sich wieder ab, während die Stimme im Hintergrund weiterspricht und die Kamera bis zur halbnahen Einstellung weiter herauszoomt: »God willing, he is a groom in heaven!« Mit einem Verpixeln des letzten Bildes endet das Video.

\subsection{EsToSa S (2013): Syrian Child cries at the death of his big brother *EMOTIONAL* (Kapitel 4.1.1)}

Das Video beginnt mit einer Nahaufnahme des leblosen Gesichts eines Jungen im Alter von sieben oder acht Jahren, der rücklings auf einer hölzernen Bahre aus alten Brettern liegt, von denen die Lackierung abblättert. Die Aufnahme ist leicht unscharf. Die Lichtverhältnisse sind gedämpft wie im Innern eines Gebäudes ohne künstliche Lichtquelle. Die Kamera ist seitlich und leicht über ihm positioniert und zeigt ihm im rechten Profil. Sein Mund und seine Augen sind leicht geöffnet. Am Mundwinkel und auf der Wange befinden sich dunkle braune Spuren von Dreck oder Blut. Er trägt eine hellblaue Jacke unter deren geöffnetem Reißverschluss ein weißes Shirt mit roten Streifen zu sehen ist. Seine Arme liegen nicht auf dem Untergrund, sondern führen hoch zu seinem Bauch. Die Kamera fährt langsam über das Gesicht, um es frontaler einzufangen. Die Kameraführung ist leicht verwackelt. Im Synchronton ist ein weibliches Schluchzen zu hören, das einen Namen sagt. Über das Video ist ein langsames, sanft klingendes Lied in Geigentönen gelegt. Im unteren Bilddrittel wird Buchstabe für Buchstabe einer Erklärung in weiß eingeblendet: »This is a child! What did he ever do wrong?! «Während der Einblendung schwenkt die Kamera langsam nach rechts den Körper des Jungen entlang. 
Seine unterhalb der Brust mit einem weißen Band zusammengebundene Hände und seine schwarze Jogginghose kommen ins Bild. Eine männliche Stimme ist zu hören, die etwas im ruhigen Ton sagt. Dann schwenkt die Kamera etwas schneller zurück zum Gesicht des Jungen, als zwei Hände aus dem linken Off auftauchen und mit hastigen Handgriffen das Shirt des Jungen an der Halsöffnung herunterziehen, um den Blick der Kamera für eine Schusswunde an der rechten Brust des Jungen freizulegen. Die Kamera geht ein Stück zurück und schwenkt nach rechts zu den Füßen des Jungen, als aus dem rechten Off eine verschleierte Frau, ganz in schwarz gekleidet, auftaucht. Von hinten kann man beobachten, wie sie sich über die Füße des Jungen beugt und diese zweimal zu küssen scheint. Die Schrift blendet langsam aus. Die Kamera schwenkt schnell nach links zurück zum Kopf des Jungen, der nach den Handgriffen nach rechts gefallen ist und nun auf der Seite liegt. Die Kamera filmt in einer halbnahen Einstellung, wie ein kleinerer Junge im hellen Kapuzenpullover vor der Stirn des toten Jungen steht. Das Gesicht hat er zum toten Jungen gewandt. Die Kamera beobachtet ihn von hinten. Im Synchronton sind mehrere Stimmen und ein stetes Schluchzen zu hören. Die Kamera schwenkt zurück zu den Füßen, an denen immer noch die Frau steht und sich wieder küssend über diese beugt. Dann streift sie sanft mit der rechten Hand über die Beine des toten Jungen und entfernt sich. Ein lautes kindliches Weinen ertönt. Am unteren Bildrand taucht kurz der Hinterkopf des kleineren Jungen auf. Er streckt seinen linken Arm nach dem toten Jungen aus. Dann wendet er sich schnell zur Frau. Am rechten Bildrand ist abgeschnitten zu sehen, wie die Frau den kleineren Jungen in den Arm nimmt. Eine aufgeregte Frauenstimme spricht. Eine weitere Einblendung erscheint: »Look at his little brother... he is devasted.« Mit dieser Einblendung schwenkt die Kamera rechtsherum und zeigt von einer Position leicht oberhalb und rechts neben dem kleineren Jungen sein weinendes Gesicht in einer halbnahen Einstellung. Der kleinere Junge kämpft sichtbar mit seiner Trauer. Die Frau entfernt sich von ihm, um sich wieder über die Füße des toten Jungen zu beugen. Am Fußende der Bahre ist eine weitere dort stehende Person in schwarzer Kleidung zu erkennen. Die Kamera schwenkt noch mal zurück zu dem kleineren Jungen, der sich rechts neben die Frau stellt. Die Schrift blendet langsam aus. Die Kamera fährt um die Frau und den Jungen herum. Dabei fängt sie einen blutroten Fleck am Fuß der Bahre ein und wie die Hände der Frau die Füße des toten Jungen umfassen. Die Kamera positioniert sich am Fuß der Bahre und zoomt an das Gesicht des toten Jungen nun von der anderen Seite heran. In einer weiteren Einblendung ist zu lesen: »...he wants his big brother back « Die Kamera zoomt wieder heraus und zeigt in einer halbnahen Einstellung nun am linken Bildrand die Frau wie sie die Füße des toten Jungen umfasst und sich wieder küssend über diese beugt. Im Hintergrund sind nun zwei weitere Personen in dunkler Kleidung im Bildausschnitt erkennbar. Die Frau hebt zwischendurch klagend die Hände. Das Weinen und Klagen im Hintergrund werden intensiver. Die Einblendung blendet 
langsam aus, während die Kamera unruhig heran-, heraus- und dann wieder heranzoomt und die Frau im Bildausschnitt zeigt, wie sie nun die Hände des toten Jungen küsst und ihm über die dunklen Haare streicht. Eine weitere Einblendung erscheint: »His mum is traumatized...would you not? « Auf dem Kopf des toten Jungen liegt nun auch die Hand des kleineren Jungen, der für die Kamera durch die Frau zunächst verdeckt ist. Als die Frau sich zurücklehnt, kommt der kleinere Junge ins Bild. Eine Hand einer nicht-sichtbaren Person liegt auf seiner rechten Schulter. Weinend küsst er dem toten Jungen die Stirn, während eine Hand von hinten seine Wange umfasst und die Frau ihm über den Kopf streicht. Der kleinere Junge entfernt sich vom toten Jungen. Die Kamera schwenkt nach links und zoomt heraus. Die Frau streift in einer halbnahen Einstellung wieder über die Beine des toten Jungen. Eine weitere Einblendung erscheint: "She used to kiss him goodbye to school.« Im Hintergrund sitzt der kleinere Junge nun bei einem Erwachsenen auf dem Arm. Hinter ihnen verläuft eine lange Abmauerung, auf der in der rechten Ecke Kleidungsstücke liegen. Die Frau bricht erneut in Wehklagen aus. Sie beugt sich nun über die Oberschenkel des toten Jungen. Ihr Schluchzen ist zu hören. Sie richtet sich wieder auf und wendet sich um. Die Kamera schwenkt leicht nach oben und zeigt den kleineren Jungen auf dem Arm eines Mannes, der neben der Frau steht. Sie unterhalten sich mit jemandem. Ihre Stimmen sind hinter den Geigentönen zu hören. Am rechten Bildrand sind zwei weitere Männer hinter der Bahre an der Wand des kleinen Raumes zu sehen. Die Einblendung liest nun:»...and kiss him good-night. Wieder bricht lautes Wehklagen aus. Die Unterhaltung wird intensiver und einer der anderen Männer hat sich rechts zu dem Mann mit dem kleineren Jungen auf dem Arm und der Frau gestellt. Die Kamera bleibt dabei die ganze Zeit auf den Körper des toten Jungen fokussiert, der mit den Füßen voran diagonal in voller Länge im Bildausschnitt liegt. In der linken unteren Bildecke fasst die Frau mit ihrer linken Hand wieder die Füße des Jungen. Das Video endet.

\subsection{SNN Shaam English News (2013b): SNN I Syria | Dar'aa | Father Refuses To Believe Son Is Dead I Oct 8, 2013 | 18+ ONLY (Kapitel 4.1.1)}

Vor einem schwarzen Hintergrund steht in weißer Schrift: »18+ Adults Only Warning - Contains Graphic Images - Not for - Documentary Evidence of Crimes Against Humanity Committed by Syrian Dictator Bashar Assad.« Nach vier Sekunden wechselt das Bild plötzlich: Die Kamera filmt von oben herab in einer halbnahen Einstellung frontal einen Mann, der auf grauem Erdboden sitzt. Vereinzelte Trümmer liegen herum. In seinem Schoß liegt regungslos ein Junge, ca. acht bis zehn Jahre alt, in kurzer Hose und kurzem Shirt. Das Shirt ist über dem Bauch zerrissen. Beine und Arme des Jungen sind blutverschmiert und verdreckt. Mit 
der linken Hand hält der Mann den Kopf des Jungen umfasst, der an seiner linken Schulter ruht. Das Gesicht des Jungen ist ebenfalls leicht mit Blut verschmiert. Die Schädeldecke des Jungen liegt offen. Am oberen Hinterkopf hängt der Haarschopf blutig herab. Um den Mann herum stehen vier Personen, von denen nur die Beine und am rechten Bildrand eine Hand zu sehen sind. Die Lichtverhältnisse sind schattig, die Aufnahme leicht unscharf. Im Synchronton ist eine laute, aufgeregt sprechende Stimme zusammen mit der Stimme des sitzenden Mannes zu hören, der den Jungen adressiert. Am unteren Bildrand wird mit einer Einblendung übersetzt: »Father: Son, where have you gone and left me. Cameraman: September 30, 2013. City of Tafas." Der Mann wendet seinen Blick an ein Gegenüber im rechten Off. In der Einblendung liest sich: „Father: My son! My son! Camerman: Casuality of rocket bombardement. Man: Seek help from God.«Die Kamera bewegt sich leicht nach links um den Mann mit dem toten Jungen im Schoß herum und erfasst den Kopf des Jungen frontaler: In einer diagonalen Linie vom rechten Wangenknochen, über den Nasenansatz hinweg bis hinauf zur Stirn ist der Schädel aufgerissen. Der Mann fährt in seinem Wehklagen fort. In der Einblendung liest sich weiter: "Father: I swear, what did he do? My son! Camerman: We have relied on God and He is the best of caretakers." Der Mann hebt den Kopf des Jungen leicht an. Kurzzeitig erscheint der leere, blutige Schädel im Bildausschnitt, bevor von rechts zwei Arme den vorderen Bildbereich füllen und den Mann an Schulter und linkem Unterarm fassen. Der Mann versucht die Schädeldecke des Jungen vergeblich zuzuklappen. Ein Beistehender legt die Hand beruhigend unter das Kinn des Mannes. Auch hinter dem Mann kommt ein weiterer Beistehender auf den Mann $\mathrm{zu}$ und versucht ihn zu beruhigen. In der Einblendung liest sich: »-We have relied on God that is sufficient for us. Father: My son, for God's sake! Cameraman: May God have revenge on you, Bashar Assad!« Aus dem rechten Off greift ein Mann direkt vor der Kamera nach links. Die Einblendung liest: »-We have relied on God and $\mathrm{He}$ is the best of caretakers. Man: Please, Sir, give him to us. Put him down, put him down. « Der Mann im Vordergrund wendet sich zu dem Mann mit dem toten Jungen im Schoß um, greift den Jungen an den Füßen und beginnt zu ziehen. Von rechts kommen weitere Männer hinzu. Einer greift den toten Jungen unter den Armen und versucht den Jungen von dem Schoß des wehklagenden Mannes zu nehmen. Die Kamera ist ein Stück zurückgegangen und zeigt nun unten rechts ein rötliches Tuch auf dem Boden liegen. Der wehklagende Mann beugt sich schützend über den toten Jungen und drückt sich an ihn. In der Einblendung liest sich: »Father: No, for God's sake, no!« Immer mehr Männer versuchen dem wehklagenden Mann den toten Jungen fortzunehmen. »Man: Sir, please, that's enough.« Der wehklagende Mann drückt den Jungen mit beiden Armen fest an sich. »Father: My love! My love, Layth! -Man: Please calm down, remember God.« Schließlich lässt der Wehklagende nach und öffnet seine Arme. Zwei Männer legen den toten Jungen an Füßen und Armen auf das ausgebreitete Tuch auf dem Boden und schlagen 
ihn darin ein. Die Kamera schwenkt mit ihnen nach rechts. In der Einblendung liest sich: »Father: My love, for God's sake. My heart! He's my life! « Die Kamera schwenkt wieder nach links zurück zum Vater, der immer noch wehklagend auf dem Boden sitzt. Mehrere Männer stehen um ihn herum und legen ihre Hände auf seine Schultern. In der Einblendung liest sich: »Man: Sir, please, remember God.« Der Wehklagende beginnt sich aufzurichten und das Video endet.

\subsection{EAU ARGENTÉE (2013) (F, R: Mohammed/Bedirxan): État de Siège (Kapitel 4.1.1)}

In einer halbnahen Einstellung sitzt eine verstümmelte, hellbraune Katze auf einer mit Schutt bedeckten Straße. Das Licht ist leicht gedämpft im Schatten von Gebäuden. Im Synchronton sind leise Bewegungen auf dem körnigen Untergrund und immer wieder entfernte Schüsse zu hören. Das linke Vorderbein der Katze ist abgerissen, ihre linke Gesichtshälfte leicht verkrustet und verdreckt. Mit einem deutlichen Mauzen humpelt sie auf die Kamera zu, die sich statisch auf ihrer Höhe befindet. „Guten Tag«, sagt Bedirxan im Off (mit deutschem Untertitel). Die Katze kommt ganz nah an die Kamera heran und richtet ihren Blick erwartungsvoll in die Kamera. »Havalo...«, fährt Bedirxan fort, während die Katze sich wieder setzt und die Kamera ihr verstümmeltes Bein fixiert. Schnitt. Die Katze humpelt ein Stück mit der Kamera die Straße entlang. Die Kamera befindet sich immer noch auf ihrer Höhe. Der Bildrahmen ist in einer Naheinstellung leicht nach links gekippt. »Homs heute...«, kommentiert Bedirxan (mit deutschem Untertitel). Der Blick der Katze lässt die Kamera nicht los. Katze und Kamera bleiben stehen. »Unser niedergebranntes Haus, die Steine«, sagt Bedirxan (mit deutschem Untertitel). Die Katze wendet den Kopf kurz nach links suchend in die Ferne, dann auf den Boden als Schüsse im Hintergrund ertönen. Die Katze dreht erst ihre Ohren nach rechts, dann wendet sie auch den Kopf nach rechts in die Richtung, aus der die Schüsse anscheinend zu hören waren. Schnitt. Die Kamera fängt in einer statischen halbtotalen Einstellung die Häusergasse ein, in der die Katze sich befindet. Der ganze Boden ist mit grauen Trümmern bedeckt. In einiger Entfernung zur Kamera hockt eine unscharfe menschliche Gestalt auf dem Boden zur Kamera gewandt. Die verstümmelte Katze säumt den linken Bildrand, den Kopf zur unscharfen Gestalt gerichtet. Die Kamera befindet sich weiterhin auf Höhe der Katze und blickt mit ihr in Richtung der unscharfen Gestalt. »die herumliegenden Sachen...«, fährt Bedirxan fort (mit deutschem Untertitel). Ein weiterer Schuss ertönt. Die Katze dreht den Kopf zur Kamera. Der obere Bildrahmen schneidet ihr Gesicht oberhalb der Schnauze ab. »Die Leichen an jeder Ecke, als Erinnerung und die Wäsche, die an der Leine hängt...", erzählt Bedirxan weiter (mit deutschem Untertitel). Die Katze senkt ihren Kopf neugierig nach unten und streckt die Nase suchend aus. Schnitt. 
Die Kamera blickt in einer halbnahen Einstellung auf die verstümmelte Katze herab, die ihr mit erhobenem Kopf entgegenhumpelt. "Das alles hat mein Herz gebrochen«, fasst Bedirxan zusammen (mit deutschem Untertitel). Die Katze bleibt stehen, mauzt eine Aufforderung in die Kamera, während diese näher an die Katze heranzoomt. bis ihr Gesicht den ganzen Bildrahmen füllt. »Havalo...", klagt Bedirxan im Off (mit deutschem Untertitel). Die Kamera zoomt wieder langsam heraus bis zur halbtotalen Einstellung, während die Katze ihren Blick in die Kamera gerichtet hält. »Havalo, Havalo«, wiederholt Bedirxan (mit deutschem Untertitel). Die Katze setzt sich und schaut weiter, gerade aufgerichtet und erwartungsvoll in die Kamera. Fliegen schwirren um sie herum. Die Katze blickt kurz nach hinten. Im Off ertönt ein heller Geigenton. »Homs, die aufgeschlitzte Stadt...«, beendet Bedirxan aus dem Off (mit deutschem Untertitel). Die Katze dreht ihren Kopf zurück und senkt ihren Blick erst nach unten, um dann wieder die Kamera zu fixieren. Schnitt.

\subsection{EAU ARGentéE (2013) (F, R: Mohammed/Bedirxan): Miaou (Kapitel 4.1.1)}

Auf die Titeleinstellung Miaou in weißer arabischer und lateinischer Schrift auf schwarzem Hintergrund folgt im Breitbild die Großaufnahme eines kleinen Kätzchens. Es ist graugetigert mit weißer Zeichnung und gerade ein paar Wochen alt. Es hockt zusammen gekauert mitten auf der Straße im Schatten von hohen Wohngebäuden, die die Straße rahmen. Seine Ohren sind versengt, seine Augen nur zu Schlitzen geöffnet. Sein Fell ist ebenso versengt wie seine Schnurhaare und Schnauze. Die Kamera befindet sich auf seiner Höhe direkt vor ihm und fängt sein wiederholtes, klagendes Miauen in einer Großaufnahme ein. Das Kätzchen richtet den fast blinden Blick rechts an der Kamera vorbei. Im Hintergrund erstreckt sich ein Straßenzug mit hohen Gebäuden, deren Füße von zusammengeschobenen Trümmerhaufen gesäumt werden. »Hast du Schmerzen?« fragt Bedirxan im Synchronton hinter der Kamera (mit deutschem Untertitel). Das Kätzchen wendet schnell seinen Kopf zu ihrer Stimme und stößt ein drängendes Miauen aus. Ausblende.

\subsection{GazpromFan 18 (2013a): 18+not for shock!) Headshot! Syrian sniper kills a terrorist of CIA FSA (LIVE) (Kapitel 4.1.2)}

Ein junger Mann in einem dunklen T-Shirt und einer dunklen Hose steht mit einer Panzerfaust auf der Schulter in einer halbnahen Einstellung an einem offenen Fenster im Innern eines Hauses. Er begibt sich in Schussposition. Ein zur Seite ge- 
schobener Fenstervorhang und ein als Ablagefläche dienender Wäscheständer weisen den Raum als ehemaligen Wohnraum aus. Am unteren Bildrand wird eine gelbe Bildunterschrift in Arabisch eingeblendet. Der Filmende befindet sich hinter einem Mauervorsprung links hinter dem Mann mit der Panzerfaust. Die Einstellung ist verwackelt, unscharf und überbelichtet. Der Mann mit der Panzerfaust lehnt sich etwas aus dem Fenster. Ein lauter Schuss ertönt und der Mann lehnt sich wieder zurück. Im Hintergrund ruft eine männliche Stimme etwas auf Arabisch und entfernte unverständliche Stimmen aus dem Innern des Hauses sind zu hören. Dann lehnt sich der Mann wieder aus dem Fenster und feuert die Panzerfaust ab. Hinten aus der Panzerfaust schießt die Verdämmung heraus. Zur selben Zeit fliegt explosionsartig etwas Nicht-Bestimmbares vom Hinterkopf des Schützen fort. Sofort sackt der Schütze in sich zusammen. Nach einem Schnitt wiederholt sich der Moment des Abschusses zweimal. Dann schließt eine Effektblende an, mit der der Augenblick, in dem hinten die Verdämmung ausgestoßen wird und zur selben Zeit am Hinterkopf des Schützen etwas wegfliegt, in Zeitlupe und vor der Einspielung eines Ausschnitts des Titelliedes des Clint-Eastwood-Westerns Für EIN PAAR DOLLAR MEHR (1965) (I/S/D, R: Leone) wiederholt wird. Dann wird dieser Augenblick in Zeitlupe rückwärts abgespielt, so dass der Mann sich wiederaufrichtet. Daraufhin wird die Szene wieder abgespielt und der Mann sackt erneut in sich zusammen. Weitere Schüsse ertönen im Synchronton, während im Off der Western-Titelsong weiterläuft. Die Kamera schwenkt nach unten, zeigt die angewinkelten Beine des getroffenen Mannes, den Boden des Raumes und einen weißen Plastikstuhl, der rücklings an dem Mauervorsprung lehnt, hinter dem sich die Kamera befindet. Mit einem weiteren hastigen und wackeligen Schwenk bewegt sich die Kamera wieder nach oben und zeigt den regungslos zusammengesackten Mann mit der Panzerfaust, die auf dem Fenstersims ruht. Schnell wendet sich die Kamera um ca. 180 Grad nach rechts und blickt eine schmale Betontreppe hinunter an deren Fuß eine dunkle, unscharfe Gestalt mit einem Maschinengewehr in der Hand hockt. Mit einem weiteren Rechtsschwenk der Kamera blendet das Video mit einer Effektblende schwarz aus und in großer gelber Schrift erscheint ein »BYE BYE«, das den ganzen Bildrahmen ausfüllt. Währenddessen ist der Western-Titelsong weiter zu hören. Das Video endet mit einer letzten Effektblende und dem Label des YouTube-Kanals Syria Tube, das unter schrillem Ton und aufwendig animiert ins Bild hineinfliegt. 


\subsection{SyrRevoDiary English (2013): Leaked video showing Shabiha playing with the corpse of a Free Syrian Army soilder. 18+ (Kapitel 4.2.1)}

Auf einer Schrifttafel, die der syrischen Staatsflagge der Syrischen Republik nachempfunden ist, ${ }^{1}$ wird unter dem Videotitel das Video mit den Worten beschrieben: "The Free Deir Ezzor station aired this video which they received from the Saraya Al Nasir Batallion. The video was found with one of the Shabiha, and it depicts them playing with the corpse of a Free Army soilder. 18+«. Darunter steht in großer Schrift: »Message to the world from Syrians«. In der nächsten Zeile liest sich: »fromsyrians.com«. Eine schwarze Einstellung folgt. Nach wenigen Sekunden setzt das Videobild ein. Das Bild ist stark verpixelt und unscharf. In der linken oberen Bildecke, weit ins Bild hineingerückt, befindet sich ein Logo im arabischen Stil. Nur schwer lassen sich in der linken Bildhälfte zwei militärisch bekleidete Männer und in der rechten ein auf dem Boden liegender, nackter und blutiger Körper ausmachen. Die Männer scheinen zu knien. Die Kamera filmt von einer Position leicht oberhalb der Männer, vielleicht aus einer stehenden Position. Die Tonqualität ist schlecht und leicht verzerrt. Mehrere Männer sprechen mit- oder durcheinander. Der nackte, blutige Körper wird bewegt, während sich die Kamera ebenfalls unruhig bewegt. Ein Stiefel kommt etwa auf Höhe des Kopfes mit einer schnellen Bewegung kurz ins Bild, als würde der Kopf getreten werden. Das Gesicht des toten Körpers erscheint in einer Nahaufnahme im Profil: Es ist ein junger Mann mit leblosem, geöffnetem Mund und Blutspuren auf der Wange und unter der Nase. Am unteren Bildrand wird in weißer Schrift eingeblendet: »This son of a bitch." Aus dem rechten Off tritt ein militärischer Stiefel mehrmals auf das Gesicht des Toten. Die Kamera schwenkt unruhig nach links und verliert das Gesicht aus dem Fokus. In der Bildunterschrift liest sich: »This dog wants freedom $\aleph^{2}$, während das Bild in großen Pixeln wechselnder Farbtöne im graubraunen, grünen und weißen Bereich verschwimmt. Die Kamera schwenkt nach rechts und findet den Toten wieder: Er wurde hochgenommen. Sein Kopf ist zur Kamera gerichtet und hängt baumelnd herab. »This is freedom«, liest die Bildunterschrift, während die männlichen Stimmen im Synchronton mehrmals dieselben Worte vermutlich in Arabisch wiederholen. Die Bildunterschrift wechselt: »This is what he asked for.«

Die Flagge mit einem grünen, einem weißen und einem schwarzen Querstreifen und drei roten Sternen auf dem weißen Querstreifen war von 1932-1958 und von 1961-1963 vor Machtergreifung der Baath-Partei und des Assad-Regimes die offizielle Staatsflagge Syriens. Vor allem in der Frühphase des Syrienkonflikts war diese häufig im Zuge von Straßenprotesten und -kämpfen zu sehen. Für weitere Informationen siehe auch Ansar 2012.

2 Dies ist als eine Referenz an die Sprechchöre während der Straßenproteste im frühen Syrienkonflikt zu sehen. Vgl. Kapitel 3.2. 
Die Kamera schwenkt unruhig hin und her. Dabei liest sich in der Bildunterschrift zuerst: »We are Assads beasts! « und dann »God is great (being said mockingly of the rebels)«. Mit einem Schwenk nach oben fängt die Kamera einen Soldaten in einer Naheinstellung ein, der unverhältnismäßig klar zuerkennen ist: Er trägt einen Einsatzhelm, militärische Einsatzkleidung und steht leicht vorgebeugt. Er blickt geradewegs in die Kamera, ruft etwas aus und richtet sich auf. In der Bildunterschrift liest sich erneut: »We are Assad's beasts! « Die Kamera schwenkt nach links und fängt einen weiteren Mann in derselben Bekleidung in einer Naheinstellung ein, der die rechte Hand wie zum Gruß nach oben hebt und ebenfalls etwas ausruft. Die Kamera schwenkt nach unten und nach rechts und zeigt erneut den Toten, wie er nun über den Rücken eines stehenden Soldaten herabhängt. Wieder liest sich: »This son of a bitch!« Die Kamera macht einen unruhigen Schwenk auf einen Haufen von Kleidung und blutigen Flecken. Vielleicht sind es weitere tote Körper? Die Bildunterschrift liest: "God is Greater than our enemy! (being said mockingly of the rebels)«Wieder bewegt die Kamera sich unruhig und fängt das gleiche Bild erneut ein, auf dem nun klarer ein auf dem Bauch liegender Toter zu erkennen ist, von dem nur der Oberkörper mit ausgestreckten Armen und mit dem Kopf nach unten gerichtet im Bildausschnitt zu sehen ist. Von links greift jemand an den Rücken des Toten und beginnt leicht an ihm zu rütteln. »Look at this sight, my ass «, steht in der Bildunterschrift. Die Kamera schwenkt um $180^{\circ}$ Grad herum und zeigt in einer Großaufnahme das Gesicht des filmenden Soldaten, der in die Kamera spricht. In der Bildunterschrift wird übersetzt: »This is just a candid shot for those who want freedom. We're going to spread this through bluetooth soon.« Während er spricht haut ihm jemand von rechts leicht gegen den Helm. Hinter ihm, über die linke Schulter hinweg, ist der Kopf eines Behelmten zu sehen. Die Kamera schwenkt noch einmal nach rechts und produziert verpixelte, unscharfe und $\mathrm{zu}$ nahe Bilder. Das Video endet.

\subsection{ForMotherSyria (2013): +21 Al Qaedac rebel in Syria, rips out the heart of a Syrian and eats it. (Kapitel 4.2.2)}

Auf schwarzem Hintergrund steht in großen roten Buchstaben »WARNING - This video contains graphic material.« In der linken oberen Bildecke ist ein Logo im Look der syrischen Flagge zu sehen, auf der eine comichafte Soldatenfigur ohne Gesicht ragt. Daneben steht in gelber Schrift: »SYRIAN SOLdier«. Das Logo bleibt das Video über in der Bildecke stehen. Nach drei Sekunden wechselt die Schrift vor schwarzem Hintergrund zu einem längeren Text. In der ersten Zeile erscheint in rot: »This is what you would not see on TV. « Darunter folgt in weiß: »An unprecedented and unreasonable Criminality reaches to cannibalism, committed by the name of the revolution, freedom and religion. This video shows one of al-Qaida 
and FSA terrorists in Syria, ripping out the heart of a Syrian Army soldier, and then he eats it. This is the freedom that the West wants in Syria, and those are the savage animals whom the Syrian Arab Army fights against, and tries to protect us from their terror.« Dann folgt eine letzte Zeile in rot: »EXPOSE THE TRUTH.« Nach zehn Sekunden setzt leise Hintergrundmusik ein. Nach 18 Sekunden wird schließlich zum angekündigten Video gewechselt: In einer halbtotalen, statischen Einstellung steht ein Mann in militärischer Kleidung seitlich mit der rechten Körperseite zur Kamera gewandt und zum Boden vorgebeugt inmitten von Trümmern, die den gesamten Hintergrund ausfüllen. Das Video ist unscharf und verpixelt. Die Farbgebung ist in den Rotbereich verschoben. Die Hintergrundmusik ist verstummt. Unter dem Mann liegt ein undeutlicher, regungsloser Körper ebenfalls in militärischer Kleidung. Rote Flecken sind auf diesem auszumachen. Der Mann ist mit beiden Händen am Körper am Hantieren, während im Synchronton eine männliche Stimme etwas sagt. Über das Bild sind neben dem einen, noch ein weiteres Logo und eine Inschrift gelegt. In der rechten Bildecke, weit ins Bild gerückt, ist das Logo von Syrian:Truth zu sehen. Am unteren Bildrand liest sich in weißer Schrift: »FB.com/Syrian.Truth«. Der Mann hebt die linke Hand etwas an. Darin hält er etwas Rötliches, das zum unter ihm liegenden Körper herunterhängt. Mit der rechten Hand führt er mit einem Gegenstand daran schneidende Bewegungen aus. Am unteren Bildrand erscheint eine Übersetzung in weißer Schrift: »You cut like a Love Heart (sarcasm). « Die Kamera schwankt leicht hin und her, während der Mann weiter schneidet und nun versucht etwas von dem rötlichen Stück abzutrennen. Am rechten Bildrand erscheint kurz ein Teil eines Körpers in khakifarbener Kleidung. Wieder ertönt eine männliche Stimme im Synchronton. In der Bildunterschrift liest sich: "I swear we will eat your hearts and livers you soldiers of Bashar. « Der Mann richtet sich auf und blickt in die Kamera. In der linken Hand hält er ein fleischfarbenes Stück des Körpers und in der rechten den Schneidgegenstand, der ebenfalls eine Rosafärbung hat. Er hebt den rechten Arm zu einer Ansprache und spricht direkt in die Kamera. Die Kamera zoomt bis zur Naheinstellung heran. Das Gesicht bleibt verpixelt, während der Mann ruft: »Takbeer! « Im Hintergrund stimmen mehrere männliche Stimmen zu der Lobpreisung an: »Allahu Akbar!«In der Bildunterschrift liest sich entsprechend: »TAKBEER: ...ALLAHUAKBAR!« Die Kamera zoomt schnell bis zur halbnahen Einstellung heraus. Die Bildunterschrift wechselt: »We are the heroes of Baba Amr We rip out the soldiers hearts and eat them." Der Mann hebt demonstrativ das fleischige Stück in seiner linken Hand, führt es zum Mund und beißt hinein. Das Video endet. 


\subsection{Deek Jackson (2014): FILMED TO DEATH IN SYRIA, UKRAINE, EGYPT, IRAQ, AFGHANISTAN ETC bla bla. (Kapitel 4.2.3)}

In einer Naheinstellung steht ein Mann mit Halbglatze, kurz geschnittenen Haaren und getrimmtem Vollbart vor einem Vollbild mit einem Landkartenausschnitt, der den Umriss Syriens zeigt. Der Mann, dessen Name, Deek Jackson, unten in einer Schriftleiste gemeinsam mit dem Titel der satirischen Nachrichtensendung, FKN NEWZ, eingeblendet wird, trägt einen schwarzen Anzug mit weißem Hemd, schwarzer Krawatte und eine schwarze Sonnenbrille. Er moderiert im Nachrichtenstil: "In Syria, the ongoing martyr and suppression of anti-Assad protests has resulted in soaring viewing figures and ratings for news shows. « Das >Screenbild wechselt zu einer verwackelten Videoaufnahme eines Amateurvideos einer Straßenszene aus der syrischen Stadt Homs (der Name wird im oberen linken Bildviertel eingeblendet), wie man es von Videos auf YouTube und Nachrichtensendungen gewohnt ist. Hinter der Schriftleiste, die nun die Worte »War is peace« anzeigt, ist das gelbe Logo des arabischen Nachrichtensenders al-Jazeera zu erkennen. Deek Jackson fährt fort: "Millions of millions of people have now watched the slaughter and have seen the dead. Charities and humanitarian groups have called for a ceaseviewing so that the eyes of many more innocent viewers can be spared.« Mit einer Effektblende wird $\mathrm{zu}$ einem Hintergrundbild mit einem Landkarten-3D-TunnelEffekt in Blautönen gewechselt: "In spite of this, broadcasts go on nightly...«, in der Schriftleiste werden nun die Worte »Freedom is slavery« eingeblendet. Mit einer weiteren Effektblende wechselt die Einstellung im Vollbild zu einer Aufnahme eines Plakats von Bashar al-Assad, das auf einem großen Aufsteller an einer Hausfassade angebracht ist. Deek Jackson moderiert aus dem Off weiter: »... with President Assad blaming international >tellevisiom « [das Wort wird wie eine Mischung aus >terrorism < und stelevision < ausgesprochen; $\mathrm{MM}$ ] for the rise in viewing figures. However, opposition groups...«, eine Effektblende bringt Deek Jackson zurück auf den Bildschirm und die Schriftleiste zeigt die Worte »Ignorance is strength « an, »... say that state-sponsored television has itself filmed many people and shot loads of footage in the outlined districts of Syria. " Eine weitere Effektblende bringt ein verwackeltes Amateurvideo auf das Hintergrundbild, das einen regungslosen Körper auf der Ladefläche eines Transporters zeigt, dessen Torso von einem hellen Tuch umhüllt ist. Wieder ist das al-Jazeera-Logo hinter der Schriftleiste zu erkennen. "The city of Homs has been filmed mercilessly by the Syrian media.« Im Hintergrund schwenkt das Video nach rechts auf zwei Männer, die einen leblosen Körper am Transporter vorbeitragen. »Some reports suggest hundreds of innocent women and children have been filmed and their images viewed around the world. Here is our special report. «Schnitt. Ein Amateurvideo eines Straßenkampfes mit Polizeieinsatzkräften und kleineren Feuern auf der Straße wird im Vollbild eingeblendet. Die Aufschrift auf den Schilden der Einsatzkräfte in griechischen und lateinischen 
Buchstaben deutet daraufhin, dass diese Szenen nicht aus Syrien stammen. Die Schriftzeile wechselt zu den Worten »Shop for victory«. Mit leicht verstellter Stimme ertönt Deek Jackson aus dem Off: »Death and suffering have always made good footage for news organizations. It no longer matters, who is dying...«, das Vollbild wechselt zu einem anderen Videoausschnitt ebenfalls von einer Straßenkampfszene vor einer ähnlichen Kulisse, »...or why. Just as long as people watch. And watch they do. Ratings for the Syrian uprising had been increasing steadily since Arab legal observers have left the country and the UN has called for the said to stop the filming and return to normal programming. «Die Straßenkampfszene wird immer gewaltvoller: Rauchbomben und Molotowcocktails werden geworfen. Maschinengewehrfeuer ist zu hören. »Distraught survivors of shelling and ...", die Schriftleiste wechselt $\mathrm{zu}$ den Worten »spend for your lives «, "... the maimed corpses of children make really good news. And western journalists have been filming in large numbers. " Ein weiterer Videoausschnitt zeigt aus nächster Nähe fliehende Menschen auf einer verrauchten Straße, die an der Kamera vorbeieilen. »Some news organizations are said to be responsible for hours of footage...", in der Schriftzeile erscheinen die Worte "Die in Debt«, »... much of it uploaded to social media spreading the horror all over the Internet. « Ein weiterer Ausschnitt einer Straßenkampfszene ist zu sehen, dieses Mal auf einer mit Bäumen gesäumten Straße und mit Einsatzkräften, die von Zivilisten mit Steinen o.ä. beworfen werden. »Millions of people continue watching and doing nothing. One woman we spoke to said: ...« Eine Nahaufnahme eines jungen Mädchens wird eingeblendet, das einen breiten Verband um den Kopf gebunden hat, der ihre Stirn, ihre Augen und ihre linke Gesichtshälfte abdeckt. Auch ihre linke Schulter ist verbunden. Ihr dunkles welliges Haar ragt hinten aus dem Verband hervor. In der rechten Bildhälfte ist ein Arm zu sehen, der den Rücken des Mädchens stützt, während sie aufrechtsitzend und leicht seitlich zur Kamera gerichtet die Lippen bewegt. Deek Jackson spricht für sie die Worte: »'Why are the people watching us die? Please watch someone else die!«« Die Schriftleiste wechselt zu den Worten »Fuck the planet«. »'There are people getting killed in Gaza, Yemen, Saudi, Bahrain. There are police states and fascisms in your own countries.« Das Bild wechselt zu einem anderen Amateurvideo, das wieder eine Straßenkampfszene, nun bei Nacht und in einem anderen Setting, zeigt. ")There are millions of starving brown people dying slowly in high definition wide screen.« In der Schriftzeile liest sich wieder »Deek Jackson«. »Please! The ratings are killing us!««, endet der Einsatz des Mädchens. Deek Jackson spricht mit unverstellter Stimme weiter: "In spite of all attempts to do nothing about this lucrative new story leaks, filming goes on. And there seems there will be no end to the rising ratings and commentaries, eh, sorry, that should be...«, in der Schriftzeile steht nun "Stay asleep«, »...killing and casualties. « Mit einem Schnitt wechselt das Bild zurück zu Deek Jackson, der vor dem Landkarten-3D-Tunnel-Effekt-Hintergrundbild mit beiden ausgestreckten, nach oben gerichteten Zeigefingern und abgewinkelten 
Daumen steht und die Zeigefinger von der Senkrechten mit einer schnellen Bewegung in die Waagerechte bringt, um in die Kamera deutend zu sagen: »Thanks, and there will be more on that story later.« Das Video endet.

\subsection{TriLingua Daniyar NAURIZ (2014): Full Uncensored Video of the James Foley Beheading (Kapitel 4.3)}

Auf einem schwarzen Bildschirm steht mittig ein weißer arabischer Schriftzug. In der linken Bildecke ist das Logo der Videoplattform Liveleak zu sehen. Nach acht Sekunden wird ein neuer Schriftzug eingeblendet. Darunter wird dieses Mal ins Englische übersetzt: »Obama authorizes military operations against the Islamic State effectively placing America upon a slippery slope towards a new war front against Muslims. «Mit einer geräuschvollen Effektblende, die das Einschalten eines Fernsehers nachahmt, setzt ein Mitschnitt einer Pressemitteilung vom 7. August 2014 des damaligen US-amerikanischen Präsidenten Barak Obama ein. Das Bild ist körnig gestört als würde es von einem alten Röhrenbildschirm stammen. Obama steht in der gewohnten Umgebung hinter einem Rednerpult, wie es aus Pressemitteilung der US-amerikanischen Regierung bekannt ist. Mit einem ernsten Gesichtsausdruck spricht Obama: "Good evening. «In einer Schriftzeile am unteren Bildrand erscheinen arabische Schriftzüge, die vermutlich Obamas Ansprache übersetzen. »Today I authorized «, fährt Obama fort, »two operations in Iraq. Targeted air strikes to protect our American personnel and a humanitarian effort to help save thousands of Iraqi civilians who are trapped on a mountain without food and water and facing almost certain death. Let me explain the actions we are taking and why: First, I said in June as the terrorist group ISIL began an advance across Iraq that the United States would be prepared to take targeted military action in Iraq if and when we determined the situation required. In recent days, these terrorists have continued to move across Iraq and have neared the city of Erbil where American diplomats and civilians serve in our consulate and military personnel advise Iraqi forces. To stop the advance on our bill I have directed our military to take targeted strikes against on ISIL terrorists' convoys if they move towards the city. We intend to stay vigilant and take action if these terrorist forces threaten our personnel or facilities anywhere in Iraq and who are in our consulate in Erbil or in our embassy in Bagdad. We are also providing urgent assistance to Iraqi government and Kurdish forces so they can move more effectively the fight against ISIL. « Mit einer erneuten Effektblende und einem knackenden Geräusch, das das abrupte Ausschalten eines Fernsehers imitiert, setzt eine kurze schwarzweiße Sequenz eines Luftschlags, aufgenommen mit einer Schießkamera, ein, die links mit einer Einblendung in arabischer und lateinischer Schrift als "American Aggression Against the Islamic State« bezeichnet wird. Im Synchronton sind Fluggeräusche und eine 
verrauschte Stimme über den Flugfunk zu hören. In der rechten oberen Bildecke befinden sich zwei schwarze, längliche Balken über dem Bild. Der Zielsucher blinkt in der Mitte des Bildes über zwei schwarzen Flecken, die sich auf einer Straße im freien Feld befinden. Mit einem Explosionsgeräusch breitet sich ruckartig ein zunächst größer-, dann kleinerwerdender weißer Fleck vom Bildmittelpunkt aus: ein Luftschlag. Die Sequenz blendet langsam aus. Das Video setzt sich mit einer als A Message to America betitelten Sequenz fort. Der Titel wird in weißen Großbuchstaben auf schwarzem Hintergrund langsam eingeblendet. Dann folgt eine langsame Überblende zu einer Wüstenkulisse. In einer halbtotalen Frontaleinstellung kniet in der Bildmitte links gerade aufgerichtet ein Mann mit kurzgeschorenem Haar und orangenem Jumpsuit auf dem Wüstenboden. An seinem Kragen unterhalb des Kinns ist ein kleines schwarzes Mikrofon angebracht. Seine Hände hält er hinter dem Rücken als seien sie dort gefesselt. Links neben ihm steht ein vermummter Mann in schwarzer Kleidung. Nur seine hellbraunen Stiefel stechen aus dem Outfit hervor. Über der linken Schulter hängt ein mittelbrauner Gurt. Er steht breitbeinig und mit locker herunterhängenden Armen da, während der Mann im orangenen Jumpsuit in die Kamera spricht: »I call on my friends, family and loved ones to rise up against my real killers,...", in einer Schriftleiste am unteren Bildrand erscheinen arabische Schriftzüge, die die Worte vermutlich übersetzen. Links darüber wird der Name des Mannes im orangenen Jumpsuit eingeblendet: James Wright Foley (darüber auch in arabischer Schrift). In der oberen linken Bildecke ist mal eine kleine wehende schwarze Flagge mit weißen arabischen Schriftzeichen, mal ein goldweißliches Logo eines arabischen Tors unterhalb des Liveleak-Logos zu sehen. Die Lichtverhältnisse sind hell und wirken natürlich. Der Schattenwurf der beiden Männer auf dem Wüstenboden deutet auf einen hohen Sonnenstand hin. Der etwas zu großwirkende Jumpsuit flattert leicht über der Bauchpartie des knienden Mannes. Neben den gesprochenen Worten sind keine weiteren Geräusche zu hören. Mit einem Wechsel zu einer nahen Profileinstellung des Mannes im orangenen Jumpsuit (am rechten Bildrand ist ein Teil des schwarzvermummten Mannes zu sehen) fährt das Statement fort: »The U.S. government. For what will happen to me is only a result of their complacency criminality. « Das Bild wechselt zurück zur halbtotalen Frontaleinstellung. »My message to my beloved parents: ...«, von links schiebt sich ein Profilfoto im >Splitscreen « ein, das einen Mann mit Sonnenbrille in der Kleidung der US-Air-Force in einer Nahaufnahme zeigt. »Save me some dignity and don't accept any legal compensation...«, im rechten >Splitscreen wechselt das Statement wieder in die nahe Profileinstellung. Im linken >Splitscreen zu einem Foto des Mannes der US-Air-Force in einer halbtotalen Profileinstellung übergeblendet. "...for my death from the same people who effectively hit the...", der >Splitscreen< schiebt sich nach links weg und das Statement ist wieder im Vollbild zu sehen, "...last nail in my coffin with their recent aerial campaign in Iraq.« Das Bild wechselt zur halbtotalen Frontaleinstellung. »I call on my brother John, 
who is a member of the U.S. Air Force: Think about what you are doing. Think about the lives you destroy including those of your own family." Das Bild wechselt zur halbnahen Profileinstellung. "I call on you, John. Think about who made the decision to bomb Iraq recently, who killed those people - who ever they may have been. Think John! Who did they really kill? Did they think about me or my family when they made that decision? I died that day, John. When your colleagues dropped that bomb on those people, they signed my death certificate. I wish I had more time. I wish I could have a hope for freedom and see my family once again, but that ship has sailed. I guess, all in all, I wish I wasn't American.« Das Bild blendet langsam aus. Mit der folgenden Einblendung erscheinen wieder der kniende Mann im orangenen Jumpsuit und der links neben ihm stehende Schwarzvermummte. Der Schwarzvermummte hat mit der rechten Hand den Knienden am Kragen des Jumpsuits gepackt und mit der rechten ein Messer mit kurzer Klinge auf ihn gerichtet, während er frontal in die Kamera spricht: »This is James Wright Foley, an American citizen of your country.« Das Bild wechselt zur halbnahen Profileinstellung, die nun auf den Schwarzvermummten fokussiert ist, der das Messer hat sinken lassen. Der Kniende hat während dessen durchgängig eine versteinerte Miene, mit zusammengekniffenen Lippen und heruntergezogenen Mundwinkeln. Das Mikrofon von seinem Kragen ist verschwunden. Der Jumpsuit flattert stärker im Wind. "As a government you have been at the forefront of the aggression towards the Islamic State. You have plotted against us and have gone far out the way to find reasons to interfere in our affairs. Today your military air force is attacking us daily in Iraq. Your strikes caused casualties amongst Muslims. You are no longer fighting an insurgency. " Der Schwarzvermummte hebt zwischendurch die rechte Hand mit dem Messer, um seine Worte zu unterstreichen. Das Bild wechselt zur halbtotalen Frontaleinstellung zurück. »We are an Islamic army and a state that has been accepted by a large number of Muslims worldwide. «Wieder wechselt das Bild zur halbnahen Profileinstellung. Auch der Saum des Oberteils des Schwarzvermummten flattert nun im Wind. "So, effectively, any aggression towards the Islamic State is an aggression towards Muslims from all roots of life who have accepted the Islamic Caliphate as their leadership. « Das Bild wechselt zur Profileinstellung. Der Schwarzvermummte hat das Messer drohend in Richtung Kamera erhoben. »So, any attempt by you, Obama, " der Schwarzvermummte lässt das Messer sinken, »to deny the Muslims their rights of living in safety under the Islamic Caliphate will result in bloodshed of your people. " In einer abschließenden Drohgebärde hebt der Schwarzvermummte das Messer noch einmal in Richtung Kamera. Dann blendet das Bild langsam aus. Mit der nächsten Einblendung tritt der Schwarzvermummte in der halbtotalen Frontaleinstellung hinter den Knienden. Er umfasst von hinten fest das Kinn des Knienden mit der linken Hand, setzt das Messer in der rechten Hand an dessen Hals und beginnt mit einer schnellen schneidenden Bewegung. Den Knienden zieht er dabei leicht nach hinten, wäh- 
rend das Bild ausblendet. Die nächste Einstellung zeigt den kopflosen Körper des James Foley längs ausgetreckt und bäuchlings auf den Boden liegend. Die linke Körperseite ist zur Kamera gewandt. Die Arme sind an den Handgelenken hinter dem Rücken zusammengebunden. Eine Blutlache ist auf dem sandigen Wüstenboden vor der Halsöffnung zu sehen. Der blutverschmierte, abgetrennte Kopf liegt aufrecht und leicht gekippt an den zusammengebundenen Händen lehnend, mit dem Gesicht in die Kamera gerichtet mittig auf dem Rücken des Enthaupteten. Die Augen sind geschlossen, der Mund zu einer leblosen Öffnung erstarrt. Die Kamera fährt in einem statischen Rechtsschwenk wenige Sekunden den leblosen Körper entlang und fängt die an den Füßen verstreut herumliegenden Sandalen ein, bevor die Aufnahme wieder ausblendet und sich die Anfangseinstellung des Statements wiederholt: In einer Frontalansicht kniet ein Mann im orangenem Jumpsuit vor einer Wüstenkulisse in einer halbnahen Einstellung mit einem regungslosen, fast grimmigen Gesichtsausdruck. Sein Name, Steven Joel Sotloff, wird links eingeblendet. Hinter ihm steht der Schwarzvermummte. Mit der rechten Hand hält der Schwarzvermummte den knienden Mann fest am Kragen seines orangenen Jumpsuits und spricht eine Drohung an den US-amerikanischen Präsidenten aus: »The life of this American citizen, Obama, depends on your next decision.« Das Bild bleibt stehen und verliert an Kontrast. Mit einer geräuschvollen Effektblende, als würde ein Röhrenfernseher ausgeschaltet werden, endet das Video.

\subsection{4 beerech (2015): ISIS Bloopers (Kapitel 4.4.1)}

Auf das Verlesen einer Filmklappe auf Arabisch begleitet durch einen englischen Untertitel »Kill and rub the infedels [sic] take 1« und das Schlagen der Filmklappe folgt eine halbtotale Frontaleinstellung eines knienden Mannes im orangenen Jumpsuit und mit hinter dem Rücken gehaltenen Händen, als wären diese dort gefesselt. Links neben ihm steht ein schwarzvermummter Mann, der über der rechten Schulter ein Maschinengewehr trägt und in der linken Hand ein Messer hält. Die Kulisse zeigt einen sandigen Abhang oder Hügel mit Grasböschungen im Hintergrund und einen wolkenverhangenen Himmel. Das Licht wirkt natürlich. Es sind keine Umgebungsgeräusche zu hören. Mit drohend erhobenem Messer spricht der Schwarzvermummte in die Kamera: »President Obama, your policy against the Islamic state - Has brought us once again«, der Schwarzvermummte senkt den Arm und zeigt mit dem Messer auf den Knienden, »to kill an American citizen.«Am unteren Bildrand wird das Gesprochene in einer Einblendung in Englisch wiedergegeben. Mit wiedererhobenem Messer fährt der Vermummte mit einem Versprecher fort: »Under those circunstamsens«. Aus dem Off berichtigt eine Männerstimme: »Circumstances! «Schnitt. Mit einem piependen Ton erscheint wieder die Filmklappe im Bild. Mit schlagen der Klappe setzt die Einstellung er- 
neut ein: »Under those circunsances.« - „CUT!« ertönt aus dem Off. Schnitt: Piep! Die Einstellung wiederholt sich: »Circum...sancnes...«, bemüht sich der Schwarzvermummte um die korrekte Aussprache. Schnitt: Piep! »Circumtancens!« Schnitt: Piep! »Circunsancens.«Schnitt: Piep! Der Vermummte hält den linken Arm mit dem Messer gesenkt, den rechten Arm hält er angewinkelt dicht am Körper und macht eine übende Handbewegung, während er versucht das Wort korrekt auszusprechen: »Circum... Circum...«. Der Kniende hat sich ihm zugewandt, blickt zu ihm herauf und spricht ihm das Wort leise vor: „Circumastances«. Der Vermummte schaut ihn an und spricht ihm das Wort Silbe für Silbe nach: „Circumastances?«. Der Kniende nickt und wiederholt ermutigend: «-Circumastances«. Schnitt: Piep! Mit überdeutlicher Betonung sagt der Schwarzvermummte in die Kamera: »Under those circumstances! «, während der Kniende leise und ermutigend nickend mitspricht. Kurz wendet der Vermummte den Blick zum Knienden, dann links an die Kamera vorbei: »I said it good. Yes?« Aus dem Off ertönt: »Continuity...«. Der Aufnahmeleiter, bekleidet mit dunklem Pullover, Jeans, weißem Schal und einem Kopfhörer, kommt von rechts in das Bild gelaufen. Der Kniende wendet sich mit einem genervten Schnalzen ab. Der Aufnahmeleiter schlägt die Hände vors Gesicht: »continuity Inaal Di...« Schnitt: Piep! Die Filmklappe kündigt den 47. Take mit arabischen Ziffern an (die arabische 4 ist spiegelverkehrt geschrieben). »Take 47. ACTION!«, tönt es aus dem Off. Die Szene startet am Anfang: »President Obama, « beginnt der Vermummte mit erhobenem Messer. Da niest der Kniende, hustet und schnieft mehrmals. Der Schwarzvermummte dreht sich zu ihm, lässt das Messer sinken, schüttelt leicht den Kopf und sagt leise auf Arabisch: »Oh my god.« Dann spricht er jemanden im Off an: »Get some Kleenex. Get some Kleenex...«, und gestikuliert mit dem Messer. Schnitt. Der Aufnahmeleiter steht rechts neben dem Knienden. Heruntergebeugt putzt er ihm die Nase. »Push«, sagt er und der Kniende schnäuzt in das Taschentuch. »Very Good«, lobt der Aufnahmeleiter, richtet sich auf und faltet das Taschentuch zusammen. Mit einer auffordernden Handbewegung ruft er ins Off: »Ismail touch up! « Schnitt. Der Tontechniker steht mit einer Mikrofonstange und Kopfhörern vor dem Knienden und dem Schwarzvermummten, der einen Schritt zurück gemacht hat. Den Rücken zur Kamera gewandt reicht der Tontechniker die Mikrofonstange dem Schwarzvermummten und öffnet eine Puderdose. Er pudert das Gesicht des Knienden unter dem prüfenden Blick des Mannes mit weißem Schal. Schnitt. Nach dem sich beide Mitglieder der Filmcrew aus dem Bild bewegt haben, fahren die Filmaufnahmen mit einem erneuten »Piep!« fort. Es folgen weitere Versprecher des Schwarzvermummten mit korrigierenden Eingriffen der Filmcrew und ausbrechendem Gelächter unter den beiden Darstellern und der Filmcrew, das noch weitere Outtakes produziert. Schließlich gelingt es dem Schwarzvermummten seinen Part korrekt aufzusagen. Dabei bewegt er sich mit einer drohenden Gebärde auf die Kamera zu und lässt den Knieenden ein Stück hinter sich zurück. Dieser schaut sich prüfend um, erkennt seine Chan- 
ce und ergreift die Flucht. Während der Kniende auf den Horizont zu rennt, bemerkt der Schwarzvermummte die Flucht und bricht mitten im Satz ab. Zuerst schaut er hinter dem Fliehenden her, dann richtet er sich ans Off: »Why doesn't he have handcuffs? « Schnitt: Piep! Der Geflohene kniet verschnaufend im Sand. Der Schwarzvermummte steht vorgebeugt neben ihm, die Hände auf die Knie abgestützt, sichtlich nach Luft ringend. Mit einem Stöhnen richtet er sich auf, stemmt die Arme in die Seiten und beugt den Rücken durch. Dann dreht er den Kopf zur Seite, hebt seine schwarze Maske an und spukt in den Sand. Schnitt: Piep! Mit erhobenem Messer spricht der Schwarzvermummte in die Kamera: »If you want the killing to stop you give us 10 million dollars, change your name to Mohammed, and kill Justin Bieber«. Schnitt. Mit einem >Allahu-Akbar<-Ausruf tritt der Schwarzvermummte hinter den Knienden. Das Messer hält er nun in der rechten Hand. Mit der linken greift er dem Knienden an den Hals und schneidet sich in den Finger. Mit einem Schmerzensaufschrei lässt er von dem Knienden ab und hält sich seine verletzte Hand. Verwundert dreht sich der Kniende zu ihm um. »Coffee! Put some coffee! Coffee! Wait...«, hört man aus dem Off, während der Aufnahmeleiter vom rechten Bildrand kurz im Bildrahmen erscheint. »Bring me coffee, bring me something...", sagt der Schwarzvermummte, während er die verletzte Hand mit der anderen haltend zwischen seine Beine drückt. »Put your hand up...put your hand up«, fordert ihn der Kniende auf. »Au!«, ruft der Schwarzvermummte aus und folgt der Anweisung. »It will stop the blooding [sic], put your hand up.« Mit einem »CUT!« aus dem Off erscheint ein verzerrtes Testbild mit einem lauten Rauschen. Schnitt. Der Schwarzvermummte steht nach Vorne gebeugt über dem Knienden, von dem nur der Torso bäuchlings auf dem Boden liegend zu sehen ist. In der rechten Hand hält er das Messer und richtet sich langsam auf. „t's [sic] a wrap! It's a wrap!« ertönt es aus dem Off begleitet von Beifall und Jubeln. Der Schwarzvermummte hebt die linke Hand zum Victory-Zeichen. Sein Zeigefinger ist verbunden. Der Aufnahmeleiter kommt klatschend vom rechten Bildrand auf den Schwarzvermummten zu, hebt die Arme dankend zum Himmel, umfasst dann mit beiden Händen das Gesicht des Schwarzvermummten und küsst ihn freudig auf die Wangen. Dann ruft er: »Allahu Akbar!«, hält die Hände vors Gesicht und schickt einen Kuss in den Himmel. Indessen kommt vom linken Bildrand der Tontechniker auf den Schwarzvermummten $\mathrm{zu}$, um diesen ebenfalls freudig zu umarmen und zu küssen. »Very good, very good «, ist aus dem Off zu hören, während der Aufnahmeleiter sich der Kamera $\mathrm{zu}$ wendet und leicht gebeugt auf diese zugeht. In einer Großaufnahme blickt er in die Kamera. Sein Gesicht verzieht sich: »Red light!«, bemerkt er. Er bewegt sich ein Stück zurück und führt mit ausgestrecktem Arm auf die Kamera deutend aus: »Oh NO! It didn't record!« Der Schwarzvermummte wendet sich ebenfalls der Kamera zu: »Didn't record? « Er beugt sich nach Vorne und blickt direkt in die Kamera: »What do you mean didn't record?« Entsetzt blickt der Aufnahmeleiter weiter auf die Kamera. Der Vermummte richtet sich auf und wendet sich an 
den Aufnahmeleiter, wobei seine blutverschmierte Hand und das blutverschmierte Messer deutlich zu sehen sind. Mit erhobenem, verbundenem Zeigefinger spricht er zu ihm: »But it's only one take! « Am linken Bildrand beugt sich der Tontechniker runter zur Kamera und ruft ebenfalls: »Didn't record «, während der Schwarzvermummte vorwurfsvoll gestikulierend den Aufnahmeleiter adressiert: "You should have record! « Die Schuld von sich weisend antwortet dieser: »Me? Record? I don't record.«»What do you mean you don't record? Than [sic] who records?!«, entgegnet der Schwarzvermummte aufgebracht. Am linken Bildrand versucht der Tontechniker sich vergeblich in die Konversation einzubringen. Dann richtet der Aufnahmeleiter seinen Blick auf ihn: »Ismail records...«. Der Schwarzvermummte dreht sich langsam zum Tontechniker um. Dieser entgegnet mit entsetzter Verwunderung und auf sich selbst deutender Handbewegung: »Ismail records? « Schnitt: Piep! Die Szene beginnt von Vorne mit neuen Darstellern: Ismail kniet mit der Mikrofonstange in der rechten Hand und einem Tongerät in der linken Hand im Sand. Neben ihm steht der Aufnahmeleiter. Er hat eine schwarze Sturmmaske über das Gesicht gezogen. In der linken Hand hält er ein Messer. Mit einer antreibenden Handbewegung sagt er zu Ismail: »Roll sound." Ismail blickt $\mathrm{zu}$ ihm hoch und nickt im leisen Ton: »Running«. Ismail packt das Tongerät in eine kleine Umhängetasche. Der Aufnahmeleiter richtet seinen Blick zur Kamera und beginnt mit erhobenem Messer: »President Obama,...«. Schnitt: Piep! Das Video endet.

\subsection{Ersterreicherr (2015): ISIS-Enthauptung mitten in WIEN I Mariahilfer Straße 21.12.2015 (Kapitel 4.4.2)}

Das Video setzt mit einer Totalen einer belebten Einkaufsstraße ein. Der Himmel ist wolkenverhangen, die Lichtverhältnisse natürlich. Die Kamera bewegt sich auf eine kleine Ansammlung vor einer Ladenzeile zu, während an ihr vorbei und vor ihr her Passanten in winterlicher Kleidung durch die Straße laufen. Viele der Passanten richten ihren Blick auf diesen, noch nicht weiter identifizierbaren Ansammlungspunkt, von dem laute, mikrofonverstärkte Rufe ausgehen: »No Border! No Nation!« Manche Passanten bleiben stehen und wenden sich um. Die Kamera drängt sich an einer Gruppe von vier Polizeibeamten vorbei, die die Ansammlung beobachten. Aus Richtung der Ansammlung ertönt laute Musik: der Chorus gefolgt von dem zweiten Vers des englischsprachigen IS-Song-Titels For the sake of Allah sind zu hören. Gleichzeitig sind undeutliche Sprechchöre, vermutlich von Passanten, zu hören. Die Kamera nähert sich langsam dem Zentrum der Ansammlung: In einer halbtotalen Einstellung knien eine Frau und ein Mann in westlicher Kleidung auf der Straße. Vor sich halten sie weiße Pappschilder mit der schwarzen Aufschrift >REFUGEES WELCOME « und drei schemenhaften Figuren, die eine fliehende Familie (Vater, Mutter und Kind) darstellen. Hinter ihnen stehen zwei vermummte 
Männer in militärischer Kleidung. Jeder hat jeweils eine Hand auf der Schulter des bzw. der vor ihm Knienden liegen. Rechts neben ihnen halten eine Frau im schwarzen Ganzkörperschleier und ein ebenfalls vermummter Mann in militärischer Kleidung ein großes Banner im Look der Flagge des Islamischen Staates zwischen sich. Ein westlich gekleideter Mann mit Brille in einer auffallend blauen Jacke reicht dem vermummten Mann am Banner von hinten ein Mikrofon. Immer wieder werden Passanten, die der Szene zu nahekommen, harsch weggeschubst. Manche Passanten nehmen die Szene mit Handys oder Kameras auf. Der Vermummte ruft mehrmals ins Mikrofon: »Allahu Akbar!«. Die Kamera schwenkt nach links und fängt mehrere Personen ein, die die Szene versuchen zu stören und von verschiedenen Männern weggeschubst werden. Die Kamera schwenkt zurück. Der Vermummte hinter dem knienden Mann hebt ein Messer an dessen Kehle. Die Kamera zoomt in eine halbnahe Einstellung. Der Vermummte imitiert eine Schnittbewegung und der Kniende fällt bäuchlings zu Boden aus dem unteren Bildrahmen heraus. Die Kamera zoomt heraus und zeigt die Szene mit dem auf dem Boden liegenden Mann, wie er regungslos das >REFUGEES-WELCOME<-Schild aufrecht und mit der Aufschrift gut sichtbar für die Kamera vor sich hält. Der Vermummte reicht dem anderen Vermummten das Messer. Während ein Passant die Szene stört und der Kamera immer wieder die Sicht versperrt, imitiert auch der andere Vermummte eine schneidende Bewegung an der Kehle der Knienden. Diese fällt ebenfalls zu Boden, das >REFUGEES-WELCOME<-Schild flach, mit der Aufschrift nach oben, vor sich liegend. Die Kamera senkt sich von der Normalsicht auf die Höhe der auf dem Boden Liegenden und verweilt dort wenige Sekunden, bis sie wieder zur Normalsicht zurückkehrt und die weiterhin hinter ihnen stehenden Vermummten zeigt. Am rechten Bildrand und im Hintergrund der Szene haben die anderen Personen ihre Positionen verlassen. Das Banner wird zusammengefaltet, während immer wieder Passanten die Szene stören. Die Kamera schwenkt wiederholt in die Menschenmenge, die sich um die Szene versammelt hat und in der es in direkter Nähe zur Szene zu kleineren Handgemengen kommt. Die Kamera hat sich ein Stück nach links bewegt und blickt nun nicht mehr frontal, sondern seitlich auf die Szene. Der Mann mit Brille in der auffallend blauen Jacke bewegt sich vom rechten Szenenrand schnell hinüber auf die linke Seite. Er bekommt das Mikrofon gereicht, während er bemüht ist einen Störenden von der Szene wegzudrängen. Die Menschenmenge wird größer, die Handgemenge um die Szene mehren sich. Überblende. Mit einem Linksschwenk blickt die Kamera von der Seite aus auf die Szene. Im Bildvordergrund stehen zwei Polizeibeamten und verdecken der Kamera teils die Sicht. Es sind immer noch viele Menschen um die Szene versammelt, die weiterhin die am Boden Liegenden mit den >REFUGEES-WELCOME«-Schildern zeigt. Die Musik des IS-Songtitels ist verstummt. Ein durcheinander an Stimmen und Rufen sind im Hintergrund zu hören. Die Polizeibeamten sind bemüht Übergriffe von Passanten auf die Szene zu verhindern. Der Mann mit Brille und der 
auffallend blauen Jacke spricht ins Mikrofon: »Wir wissen nicht, wann das nächste hier passiert. "Die Kamera geht links um die Beamten herum, um einen unverstellten Blick auf die Szene zu haben. »Wir wissen es nicht, weil kein Mensch weiß, wie viele Schläfer durch die Grenzen gekommen sind.« Die Situation bleibt unruhig. »Wir wissen es nicht, weil kein Mensch, kontrolliert hat, wer in unser Land kommt. Diese Leute, ...", der Mann hebt den rechten Arm und zeigt in die vor der Szene versammelte Menschenmenge. Die Kamera folgt mit einem Linksschwenk seiner Armbewegung und zeigt Menschen, die Sprechchöre rufen und mit Handys und Kameras Aufnahmen von der Szene machen. "... die >Refugees Welcome schreien, haben auch Terroristen...«. Überblende. Die Kamera filmt nun aus der Menschenmenge heraus frontal die Szene. Einige Menschen versperren die direkte Sicht. "Diese Leute sind schuld an der Gefahr, die wir heute in Europa haben«, spricht der Mann mit Brille und blauer Jacke weiter ins Mikrofon. Die Sprechchöre aus der Menschenmenge halten an. Zwischen der Szene und der Menschenmenge haben sich die Polizeibeamten in einer Reihe aufgestellt. Die Kamera bewegt sich langsam nach links und bleibt auf den Mann am Mikrofon gerichtet. "Sie sprechen von >Frieden folgt $<$, aber was wir sehen ist Überfremdung und Islamisierung.«Schnitt. Von einer Position weiter links zoomt die Kamera auf den Mann am Mikrofon bis zur halbnahen Einstellung heran. »Wir sind die Kraft, die österreichische Werte und Traditionen verteidigt und wir werden weitermachen, bis unsere Heimat gesichert ist.« Die Sprechchöre aus der Menschenmenge halten an. Der Mann am Mikrofon stimmt zu einem Sprechgesang an: „Festung Europa! Macht die Grenzen dicht! «Schnitt. In einer halbtotalen Einstellung ist der obere Stock eines Kaufhauses, an dessen Schaufenster zwei Männer stehen, die interessiert nach draußen auf die unter ihnen liegende Szene blicken. Die Kamera zoomt heraus und schwenkt leicht nach rechts, um den großen Menschenauflauf vor der Szene in der Innenstadt zu zeigen. Die Kamera ist nun weit links von der Szene positioniert und blickt von der Seite auf das Geschehen. Mehrere Polizeibeamte und auch einige vermummte Personen sind in der Menschenmenge zu sehen. Es bleibt laut und unruhig. Auf einmal beginnt ein Mann in direkter Nähe zu Klatschen und viele andere folgen seinem Beispiel. Schnitt. Eine belebte Straßenecke einer Einkaufsstraße. Begleitet von Polizeibeamten überqueren die Protagonisten der vorherigen Szene die Straße. Es ist ruhiger geworden. Motorengeräusche von der Straße sind zu hören. Überblende. In einer halbtotalen Einstellung sitzen drei Personen, maskiert mit Pferdeköpfen an einer Ladenecke. Im Vordergrund sind die Spitzen von zum Verkauf stehenden Tannenbäumen zu sehen. Die Pferdekopffiguren spielen alle ein Akkordeon, während Passanten mit ihren Einkaufstaschen an ihnen vorübergehen. Ausblende. 


\section{Medienwissenschaft}

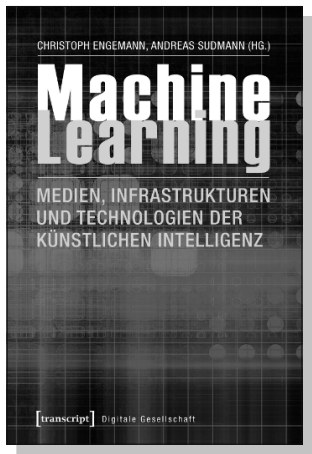

Christoph Engemann, Andreas Sudmann (Hg.)

Machine Learning -

Medien, Infrastrukturen und Technologien

der Künstlichen Intelligenz

2018, 392 S., kart.

$32,99 €(D E), 978-3-8376-3530-0$

E-Book:

PDF: $32,99 €$ (DE), ISBN 978-3-8394-3530-4

EPUB: $32,99 €$ (DE), ISBN 978-3-7328-3530-0

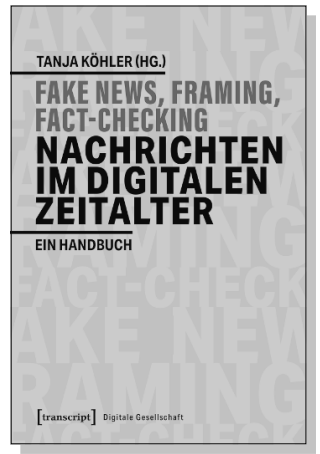

Tanja Köhler (Hg.)

Fake News, Framing, Fact-Checking: Nachrichten im digitalen Zeitalter Ein Handbuch

Juni 2020, 568 S., kart., 41 SW-Abbildungen 39,00€ (DE), 978-3-8376-5025-9

E-Book:

PDF: 38,99 € (DE), ISBN 978-3-8394-5025-3



Geert Lovink

\section{Digitaler Nihilismus}

Thesen zur dunklen Seite der Plattformen

2019, 242 S., kart.

24,99 € (DE), 978-3-8376-4975-8

E-Book:

PDF: $21,99 €$ (DE), ISBN 978-3-8394-4975-2

EPUB: $21,99 €$ (DE), ISBN 978-3-7328-4975-8 


\section{Medienwissenschaft}
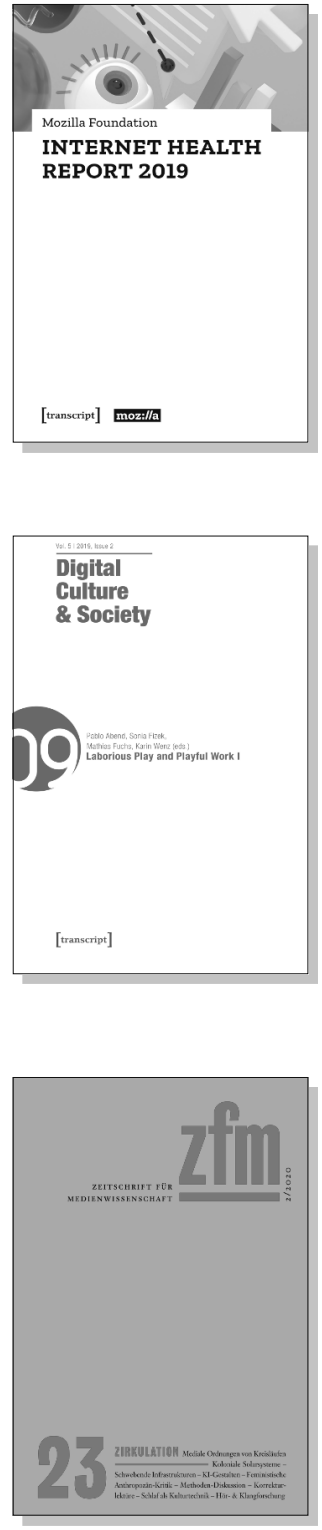

Mozilla Foundation

Internet Health Report 2019

2019, 118 p., pb., ill.

19,99€ (DE), 978-3-8376-4946-8

E-Book: available as free open access publication

PDF: ISBN 978-3-8394-4946-2

Pablo Abend, Sonia Fizek, Mathias Fuchs, Karin Wenz (eds.)

Digital Culture \& Society (DCS)

Vol. 5, Issue 2/2019 - Laborious Play and Playful Work I

September 2020, 172 p., pb., ill.

29,99€ (DE), 978-3-8376-4479-1

E-Book:

PDF: 29,99€ (DE), ISBN 978-3-8394-4479-5
Gesellschaft für Medienwissenschaft (Hg.)

\section{Zeitschrift für Medienwissenschaft 23}

Jg. 12, Heft 2/2020: Zirkulation.

Mediale Ordnungen von Kreisläufen

September 2020, 218 S., kart.

24,99€ (DE), 978-3-8376-4924-6

E-Book: kostenlos erhältlich als Open-Access-Publikation PDF: ISBN 978-3-8394-4924-0

ISBN 978-3-7328-4924-6 